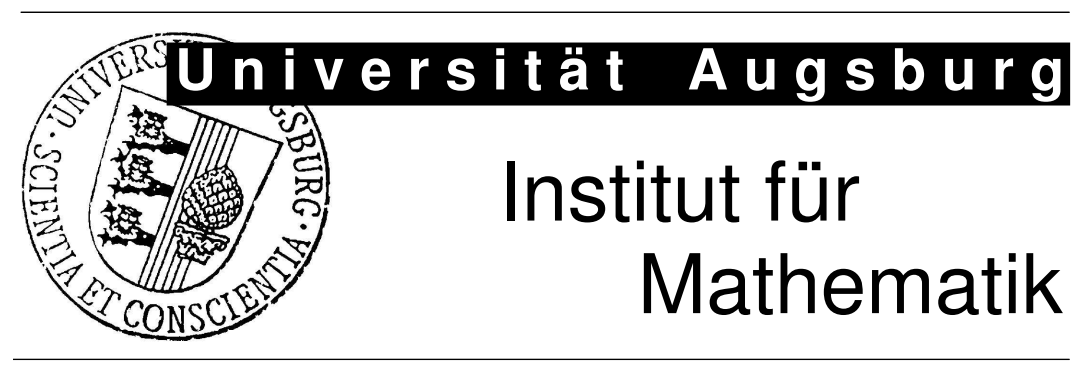

Christian Pötzsche und Martin Rasmussen

Computation of Integral Manifolds for Carathéodory Differential Equations

Preprint Nr. 26/2008 - 01. Juli 2008

Institut für Mathematik, Universitätsstraße, D-86135 Augsburg

http://www .math. uni-augsburg.de/ 


\section{Impressum:}

Herausgeber:

Institut für Mathematik

Universität Augsburg

86135 Augsburg

http://www . math. uni-augsburg.de/forschung/preprint/

ViSdP:

Martin Rasmussen

Institut für Mathematik

Universität Augsburg

86135 Augsburg

Preprint: Sämtliche Rechte verbleiben den Autoren (C) 2008 


\title{
Computation of integral manifolds for Carathéodory differential equations
}

\author{
CHRISTIAN PÖTZSCHE \\ Centre for Mathematical Sciences, Munich University of Technology, \\ 85748 Garching, Germany. \\ MARTIN RASMUSSEN \\ Department of Mathematics, University of Augsburg, \\ 86135 Augsburg, Germany.
}

We derive two numerical approximation schemes for local invariant manifolds of nonautonomous ordinary differential equations which can be measurable in time and Lipschitzian in the spatial variable. Our approach is inspired by previous work of Jolly \& Rosa (2005), 'Computation of non-smooth local center manifolds', IMA Journal of Numerical Analysis, 25, 698-725, on autonomous ODEs and based on truncated Lyapunov-Perron operators. Both of our methods are applicable to the full hierarchy of strongly stable, stable, center-stable and the corresponding unstable manifolds, and exponential refinement strategies yield exponential convergence.

Several examples illustrate our approach.

Keywords:

Invariant manifolds, Integral manifolds, Lyapunov-Perron operator, Carathéodory condition

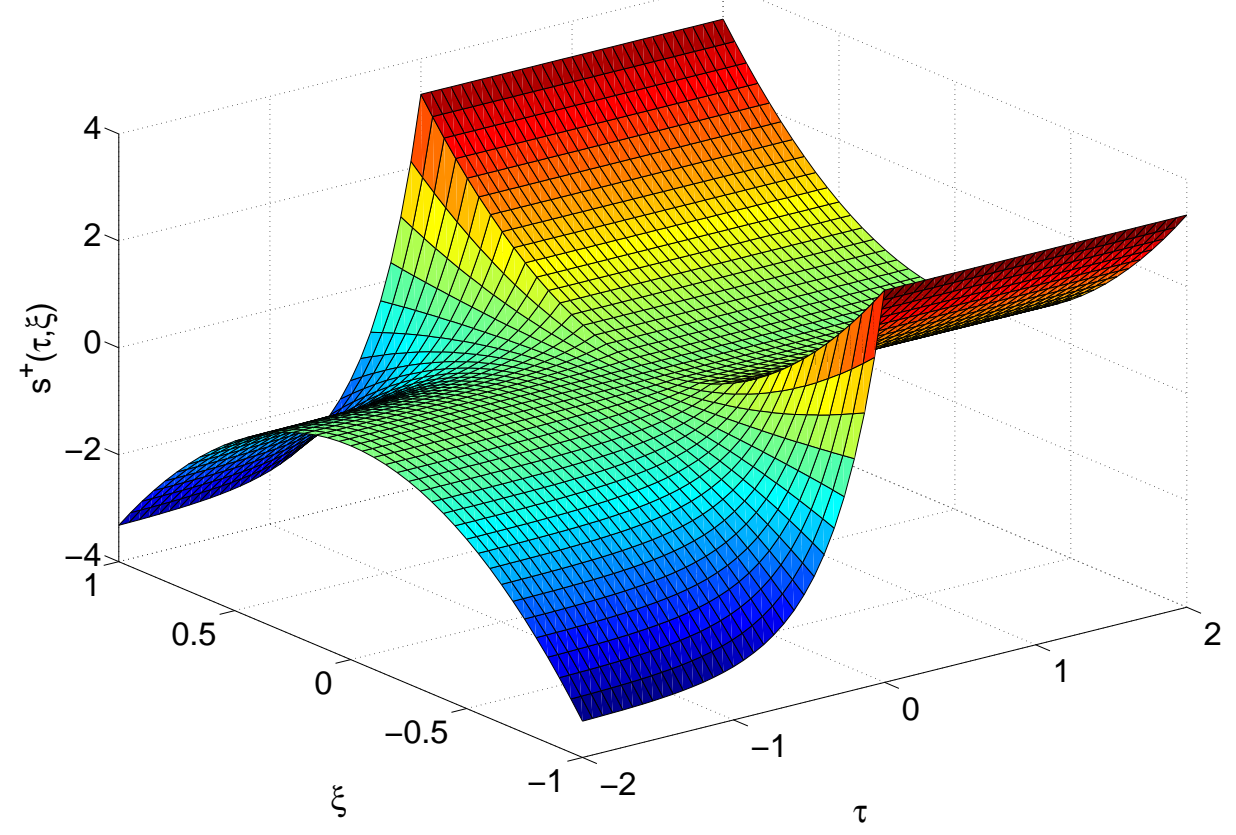




\section{Introduction}

Invariant manifolds play a fundamental role for a deeper understanding of the asymptotic behavior of nonlinear dynamical systems. In the local analysis, for instance, center manifolds are an essential tool to reduce a given system to a domain relevant for studies in bifurcation and stability theory. Concerning the global behavior, one is interested in unstable manifolds, yielding the skeleton of global attractors. Furthermore, stable manifolds frequently form the boundary for the domains of attraction in multistable systems. The computation of invariant manifolds in closed analytic form, however, is only possible in very rare cases. Hence, many different approaches to the numerical approximation have been discussed over the last years.

In this article, we propose two different numerical approximation schemes for invariant manifolds of nonautonomous Carathéodory type differential equations, i.e., we consider equations with only measurable time dependence, and in addition, we do not assume smoothness in the spatial variable, beyond being Lipschitz. Due to the weak hypotheses on the right hand side, Carathéodory differential equations provide a flexible tool in modern applications. In control theory, for instance, Carathéodory functions are used to model control inputs with values in a compact and convex set, leading to a skew product flow with a compact base set (see Colonius \& Kliemann (2000)). Particularly, switching systems have been of considerable interest for many years in the computing communities and are now intensively studied by control and systems engineers (see, e.g., Liberzon (2003)). Moreover, random differential equations driven by metric dynamical systems are path-wise nonautonomous Carathéodory type differential equations (see Arnold (1998)). Systems depending non-smoothly on their spatial variables occur, for instance, in electrical engineering (cf., Maggio et al. (2000)). As introduction to Carathéodory differential equations, we refer to the final chapter of the monograph Kurzweil (1986) or the well-written article Aulbach \& Wanner (1996).

Due to our general nonautonomous framework, the Lyapunov-Perron approach to construct invariant manifolds appears to be adequate and sufficiently flexible. It is based on a dynamical characterization as sets of trajectories with a particular asymptotic behavior. Indeed, such solutions can be classified via fixed points of an infinite integral operator (the Lyapunov-Perron operator) in a space of exponentially weighted functions, provided the Lipschitz-constant of the nonlinearity is bounded on bounded sets. For numerical purposes, one has to truncate the integral over an infinite interval by a finite, but sufficiently large one. Recently, this approach has already been examined in the context of nonautonomous difference equations or temporal discretizations of differential equations (see Pötzsche \& Rasmussen (2008)). In this reference, we formulated a numerical scheme based on the treatment of a nonlinear algebraic equation of finite dimension.

In contrast to this case, the algorithms developed in the paper at hand do not require an initial timediscretization of an ODE. As a result, the truncated continuous Lyapunov-Perron equations are Fredholm integral equations of second kind and consequently still infinite-dimensional problems. Although it is possible to apply numerical solvers for such problems directly, we propose an iterative algorithm to approximate the fixed point of the truncated Lyapunov-Perron operator in this article. Moreover, we show that this fixed point is arbitrarily close to the invariant manifold under consideration (provided the length of the truncation interval is big enough). We point out that this approach is universally applicable, including the full hierarchy of strongly stable, stable, center-stable, as well as the corresponding unstable manifolds as special cases. Our second approximation scheme generalizes results of Jolly \& Rosa (2005) to nonautonomous equations and is based on the iteration of a discrete analogue of the Lyapunov-Perron operator on piecewise constant functions under application of an exponential refinement strategy. It works without a previous truncation process and is applicable in first instance 
only to center manifolds (and some other related manifolds). The approximation of arbitrary invariant manifolds is then obtained by using appropriate nonautonomous spectral transformations. We prove an approximation result showing exponential convergence to the invariant manifold.

This paper is organized as follows. Section 2 is devoted to statement of basic facts about Carathéodory type differential equations, and we formulate our hypotheses on the linear and nonlinear part of the Carathéodory differential equation. In addition, we introduce the truncated Lyapunov-Perron operator and show that this operator admits a unique fixed point. We also summarize basic existence results on invariant manifolds for the reader's convenience. In Section 3, we show that the fixed point of the truncated Lyapunov-Perron operator yields an approximation of the invariant manifold. The quality of which depends on the length of the truncation interval and the spectral gap of the linearization, and we propose an iterative method for the approximation of this fixed point. In Section 4, we introduce our second algorithm which generalizes the work of Jolly \& Rosa (2005), and finally, the results of this paper are illustrated by means of several examples in Section 5. Among them is a 2-dimensional nonautonomous epidemic model (cf. Dushoff et al. (2004)), which is discontinuous in time, and the 3-dimensional Colpitts oscillator (cf. Maggio et al. (2000)), whose right-hand side is not differentiable in space. Clearly, when it comes to numerical implementation, a measurable time-dependence is not feasible, and we only deal with a finite number of discontinuities in the temporal variable.

We close this introduction by giving a short overview of several other methods for the approximation of integral manifolds for autonomous ODEs. Fuming \& Küpper (1994) use a discrete version of the Lyapunov-Perron operator to approximate center manifolds; Guckenheimer \& Vladimirsky (2004) apply a PDE approach to obtain global invariant manifolds, and Henderson (2005) deals with differential equations by considering whole bundles of trajectories and describing an algorithm to control them in order to approximate invariant manifolds. Moore \& Hubert (1999) explain several methods for the computation of stable manifolds of continuous systems, and they distinguish between indirect methods (which use a corresponding discrete system) and direct methods (which use the original system). Robinson (2002) uses both the construction via the graph transform and the Lyapunov-Perron operator to approximate inertial manifolds. Finally, Osinga et al. (2004) describe an algorithm for the computation of strongly unstable invariant manifolds. Various illustrative examples on this extensive area can be found in the well-written and interesting survey paper Krauskopf et al. (2005), to which we also refer for a more complete overview of the corresponding vast literature.

All of the above references deal with time-invariant equations, and thus, to our best knowledge the list of references dealing with nonautonomous equations is rather sparse. Yet, Mancho et al. (2003) derive algorithms for the computation of stable and unstable manifolds related to general hyperbolic trajectories of smooth 2-dimensional ODEs. Nonautonomous Taylor approximations of integral manifolds have been dealt with in Pötzsche \& Rasmussen (2006); in contrast to the autonomous situation, the Taylor coefficients are not obtained as a solution of an algebraic equation but as a bounded solution of a linear differential equation in the space of multilinear mappings.

Notation. As usual, $\mathbb{R}^{d}$ is the $d$-dimensional Euclidian space equipped with norm $\|\cdot\|$, and $\mathbb{R}^{d \times d}$ denotes the Banach algebra of $d \times d$-matrices. We write $U_{\varepsilon}\left(x_{0}\right)=\left\{x \in \mathbb{R}^{d}:\left\|x-x_{0}\right\|<\varepsilon\right\}$ for the $\varepsilon$-neighborhood of a point $x_{0} \in \mathbb{R}^{d}$ and abbreviate the intervals

$$
I_{\tau}^{+}(T):=\left\{\begin{array}{l}
{[\tau, \tau+T], \quad T<\infty,} \\
{[\tau, \infty), \quad T=\infty}
\end{array}, \quad I_{\tau}^{-}(T):=\left\{\begin{array}{l}
{[\tau-T, \tau], \quad T<\infty,} \\
(-\infty, \tau], \quad T=\infty
\end{array} .\right.\right.
$$

For a real number $x$, we make use of the floor function $\lfloor x\rfloor:=\max \{k \in \mathbb{Z}: k \leqslant x\}$ and the ceiling function $\lceil x\rceil:=\min \{k \in \mathbb{Z}: x \leqslant k\}$. 


\section{Carathéodory differential equations and integral manifolds}

For a nicely written introduction to the geometric theory of differential equations with measurable righthand side, we refer to Aulbach \& Wanner (1996), which will be our standard reference. All measuretheoretical terminology will refer to the Lebesgue-measure on real intervals.

We prescribe a nonempty open interval $I$ unbounded above or below. Given a locally integrable mapping $A: I \rightarrow \mathbb{R}^{d \times d}$, formally $A \in \mathscr{L}_{\mathrm{loc}}^{1}\left(I, \mathbb{R}^{d \times d}\right)$, a linear Carathéodory differential equation reads as

$$
\dot{x}=A(t) x
$$

The transition matrix of (2.1) is the unique linear mapping $\Phi(t, \tau): \mathbb{R}^{d} \rightarrow \mathbb{R}^{d}$, continuous in $t, \tau \in I$, satisfying the integral equation

$$
\Phi(t, \tau)=\mathrm{id}+\int_{\tau}^{t} A(s) \Phi(s, \tau) \mathrm{d} s \quad \text { for all } \tau, t \in I .
$$

We suppose a generalized (i.e., pseudo-hyperbolic) exponential dichotomy assumption for (2.1):

Assumption 2.1 Let $\alpha<\beta, K_{+}, K_{-} \geqslant 1$, and consider (2.1) with $A \in \mathscr{L}_{\mathrm{loc}}^{1}\left(I, \mathbb{R}^{d \times d}\right)$. We assume that there exist functions $P_{-}, P_{+}: I \rightarrow \mathbb{R}^{d \times d}$ of projections with $P_{-}(t)+P_{+}(t) \equiv$ id on $I$,

$$
\begin{aligned}
\Phi(t, s) P_{-}(s) & =P_{-}(t) \Phi(t, s) \\
\left\|\Phi(t, s) P_{+}(s)\right\| & \leqslant K_{+} e^{\alpha(t-s)} \quad \text { for all } s \leqslant t \\
\left\|\Phi(t, s) P_{-}(s)\right\| & \leqslant K_{-} e^{\beta(t-s)} \quad \text { for all } t \leqslant s .
\end{aligned}
$$

REMARK 2.1 In the autonomous case (i.e., $A(t) \equiv A_{0}$ ), the linearized system (2.2) admits an exponential dichotomy for growth rates $\alpha<\beta$, if the real part of each spectral point for $A_{0}$ does not lie in $[\alpha, \beta]$. If $A$ is $\omega$-periodic, one considers the moduli of the Floquet multipliers to obtain a similar statement. Finally, in Dieci \& van Vleck (2007), QR factorizations are used to verify exponential dichotomies for nonautonomous systems numerically.

Assumption 2.2 Suppose $F: I \times \mathbb{R}^{d} \rightarrow \mathbb{R}^{d}$ is a function satisfying the following conditions:

1. $F(t, 0)=0$ a.e. in $I$,

2. $F(\cdot, x): I \rightarrow \mathbb{R}^{d}$ is measurable for all $x \in \mathbb{R}^{d}$ (w.r.t. the Borel $\sigma$-algebras on $I$ and $\mathbb{R}^{d}$ ),

3. there exists a nondecreasing function $l:[0, \infty] \rightarrow[0, \infty]$ such that one has the Lipschitz estimate

$$
\|F(t, x)-F(t, \bar{x})\| \leqslant l(r)\|x-\bar{x}\| \quad \text { for all } x, \bar{x} \in U_{r}(0) \text { and a.e. in } I .
$$

Under these assumptions one can show that the Carathéodory differential equation

$$
\dot{x}=A(t) x+F(t, x)
$$

is well-posed in the sense that for each $\tau \in I$ and $\xi \in \mathbb{R}^{d}$, there exists a maximal open interval $J(\tau, \xi) \subseteq I$ with $\tau \in J(\tau, \xi)$ and a so-called solution $\phi: J(\tau, \xi) \rightarrow \mathbb{R}^{d}$ satisfying the integral equation

$$
\phi(t)=\xi+\int_{\tau}^{t}[A(s) \phi(s)+F(s, \phi(s))] \mathrm{d} s \quad \text { for all } t \in J(\tau, \xi)
$$


(see (Kurzweil, 1986, Theorem 18.4.2) or apply (Aulbach \& Wanner, 1996, Theorem 2.4) using cut-off techniques). Having this at hand, we denote $\varphi(\cdot ; \tau, \xi):=\phi$ as general solution of equation (2.2). In case $\sup _{r \geqslant 0} l(r)<\infty$, one has global existence of solutions, i.e., $J(\tau, \xi)=I$ for all $\tau$, $\xi$. Moreover, the function $\varphi:\left\{(t, \tau, \xi) \in I \times I \times \mathbb{R}^{d}: t \in J(\tau, \xi)\right\} \rightarrow \mathbb{R}^{d}$ is continuous and a 2-parameter group:

$$
\varphi(\tau, \tau, \xi)=\xi, \quad \varphi(t, s, \varphi(s, \tau, \xi))=\varphi(t, \tau, \xi) \quad \text { for all } \tau \in I, \xi \in \mathbb{R}^{d}, s, t \in J(\tau, \xi) .
$$

In order to provide some geometric insight, the product $I \times \mathbb{R}^{d}$ is called extended state space and a subset $S \subseteq I \times \mathbb{R}^{d}$ is called nonautonomous set with $t$-fiber $S(t):=\left\{x \in \mathbb{R}^{d}:(t, x) \in S\right\}$. Such a nonautonomous set is invariant, if $\varphi(t, \tau, S(\tau))=S(t)$ for $\tau, t \in I$. An invariant nonautonomous set $S \subseteq I \times \mathbb{R}^{d}$ is called an integral manifold of equation (2.2), if each fiber $S(t), t \in I$, is graph of a function.

Our next goal is to characterize nonautonomous sets consisting of exponentially bounded solutions of (2.2) using fixed-point arguments. With given $\gamma \in \mathbb{R}, \tau \in I$ and $T \in(0, \infty]$, the set

$$
\mathscr{X}_{\tau, \gamma}^{ \pm}(T):=\left\{\phi \in \mathscr{C}\left(I_{\tau}^{ \pm}(T), \mathbb{R}^{d}\right): \sup _{t \in I_{\tau}^{ \pm}(T)} e^{\gamma(\tau-t)}\|\phi(t)\|<\infty\right\}
$$

is a Banach space w.r.t. the norm

$$
\|\phi\|_{\tau, \gamma}^{ \pm}:=\sup _{t \in I_{\tau}^{ \pm}(T)} e^{\gamma(\tau-t)}\|\phi(t)\|
$$

Note that the set $\mathscr{X}_{\tau, \gamma}^{ \pm}(T)$ is independent of $\gamma$ for $T<\infty$. We used the abbreviation $\mathscr{X}_{\tau, \gamma}^{ \pm}(T)$ to denote one of the spaces $\mathscr{X}_{\tau, \gamma}^{+}(T)$ or $\mathscr{X}_{\tau, \gamma}^{-}(T)$ - a convenient practice applied in various contexts for the remaining paper. Moreover, one has the continuous embeddings

$$
\mathscr{X}_{\tau, \gamma}^{+}(T) \hookrightarrow \mathscr{X}_{\tau, \delta}^{+}(T) \quad \text { and } \quad \mathscr{X}_{\tau, \delta}^{-}(T) \hookrightarrow \mathscr{X}_{\tau, \gamma}^{-}(T) \text { for all } \gamma \leqslant \delta .
$$

Our overall approach is to characterize and approximate integral manifolds as a fixed point problem in $\mathscr{X}_{\tau, \gamma}^{ \pm}(T)$. Thereto, the crucial objects are Lyapunov-Perron operators $\mathscr{T}_{T}^{ \pm}: \mathscr{X}_{\tau, \gamma}^{ \pm}(T) \times \mathbb{R}^{d} \rightarrow \mathscr{X}_{\tau, \gamma}^{ \pm}(T)$,

$$
\begin{aligned}
& \mathscr{T}_{T}^{+}(t, \phi, \xi):=\Phi(t, \tau) P_{+}(\tau) \xi+\int_{\tau}^{\tau+T} G(t, s) F(s, \phi(s)) \mathrm{d} s \\
& \mathscr{T}_{T}^{-}(t, \phi, \xi):=\Phi(t, \tau) P_{-}(\tau) \xi+\int_{\tau-T}^{\tau} G(t, s) F(s, \phi(s)) \mathrm{d} s
\end{aligned}
$$

where we used the abbreviation $\mathscr{T}_{T}^{+}(t, \phi, \xi):=\mathscr{T}_{T}^{+}(\phi, \xi)(t)$ and Green's function $G$ is defined by

$$
G(t, \tau):=\left\{\begin{aligned}
-\Phi(t, \tau) P_{-}(\tau) & \text { for } t<\tau \\
\Phi(t, \tau) P_{+}(\tau) & \text { for } t \geqslant \tau
\end{aligned}\right.
$$

Proposition 2.1 Let $\tau \in I, \xi \in \mathbb{R}^{d}, \gamma \in(\alpha, \beta)$ and suppose Assumptions 2.1-2.2. If a function $\phi \in \mathscr{X}_{\tau, \gamma}^{ \pm}(\infty)$ satisfies

$$
l\left(\sup _{t \in I_{\tau}^{ \pm}(\infty)}\|\phi(t)\|\right)<\infty
$$

then the following assertions are equivalent:

(a) $\phi$ solves the Carathéodory differential equations (2.2) with $P_{ \pm}(\tau) \phi(\tau)=\xi$, 
(b) $\phi$ is a fixed point of the Lyapunov-Perron operator (2.5) resp. (2.6).

Proof. We restrict to the case $\phi \in \mathscr{X}_{\tau, \gamma}^{+}(\infty)$, since the dual situation $\phi \in \mathscr{X}_{\tau, \gamma}^{-}(\infty)$ can be treated similarly, and define $R:=\sup _{\tau \leqslant t}\|\phi(t)\|$. Let us consider functions $\phi_{ \pm}(t):=P_{ \pm}(t) \phi(t)$ for $t \in I_{\tau}^{+}(\infty)$.

$(a) \Rightarrow(b)$ If $\phi$ solves the Carathéodory equation (2.2), then $\phi_{+}$satisfies the initial value problem

$$
\dot{x}=A(t) P_{+}(t) x+P_{+}(t) F(t, \phi(t)), \quad x(\tau)=\xi
$$

and the variation of constants formula (cf. (Aulbach \& Wanner, 1996, Theorem 2.10)) yields $\phi_{+}(t)=$ $P_{+}(t) \mathscr{T}_{T}^{+}(t, \phi, \xi)$ for all $t \geqslant \tau$. Moreover, by Assumptions 2.1-2.2 and the triangle inequality, we have

$$
\|F(t, \phi(t))\| e^{\gamma(\tau-t)} \leqslant K_{-} l(R)\|\phi(t)\| e^{\gamma(\tau-t)} \leqslant K_{-} l(R)\|\phi\|_{\tau, \gamma}^{+}
$$

for all $\tau \leqslant t$, and hence, the inhomogeneous part of equation

$$
\dot{x}=A(t) P_{-}(t) x+P_{-}(t) F(t, \phi(t))
$$

is exponentially bounded. By (Aulbach \& Wanner, 1996, Lemma 3.6), this equation admits a unique solution $\phi_{-} \in \mathscr{X}_{\tau, \gamma}^{+}(\infty)$, which additionally has the form $\phi_{-}(t)=P_{-}(t) \mathscr{T}_{T}^{-}(t, \phi, \xi)$ for all $\tau \leqslant t$. Then $\phi=\phi_{-}+\phi_{+}$solves the fixed point problem for (2.5).

$(b) \Rightarrow(a)$ Conversely, let $\phi \in \mathscr{X}_{\tau, \gamma}^{+}(\infty)$ be a fixed point of the mapping (2.5). Then the variation of constants formula implies that $\phi_{+}$is the unique forward solution of the initial value problem (2.7). Furthermore, again (Aulbach \& Wanner, 1996, Lemma 3.6) guarantees that $\phi_{-}$is an exponentially bounded solution of the linear inhomogeneous system (2.8).

Proposition 2.2 Let $\tau \in I, \xi \in \mathbb{R}^{d}, T \in(0, \infty]$, suppose Assumption 2.1-2.2 with

$$
\ell:=\left(K_{-}+K_{+}\right) L<\frac{\beta-\alpha}{2}, \quad L:=l(\infty)
$$

and choose a real number $\sigma \in\left(\ell, \frac{1}{2}(\beta-\alpha)\right]$. Then, for $\gamma \in[\alpha+\sigma, \beta-\sigma]$ the Lyapunov-Perron operator $\mathscr{T}_{T}^{ \pm}: \mathscr{X}_{\tau, \gamma}^{ \pm}(T) \times \mathbb{R}^{d} \rightarrow \mathscr{X}_{\tau, \gamma}^{ \pm}(T)$ is a contraction in the first argument with

$$
\left\|\mathscr{T}_{T}^{ \pm}(\phi, \xi)-\mathscr{T}_{T}^{ \pm}(\bar{\phi}, \xi)\right\|_{\tau, \gamma}^{ \pm} \leqslant \frac{\ell}{\sigma}\|\phi-\bar{\phi}\|_{\tau, \gamma}^{ \pm} \quad \text { for all } \phi, \bar{\phi} \in \mathscr{X}_{\tau, \gamma}^{ \pm}
$$

Moreover, its unique fixed point $\phi_{T}^{ \pm}(\xi) \in \mathscr{X}_{\tau, \gamma}^{ \pm}(T)$ does not depend on $\gamma$ and satisfies

$$
\left\|\phi_{T}^{ \pm}(\xi)\right\|_{\tau, \gamma}^{ \pm} \leqslant \frac{K_{ \pm} \sigma}{\sigma-\ell}\left\|P_{ \pm}(\tau) \xi\right\| .
$$

Proof. Let $\tau \in I, \xi, \bar{\xi} \in \mathbb{R}^{d}$ and $\phi, \bar{\phi} \in \mathscr{X}_{\tau, \gamma}^{ \pm}(T)$. The proof is conceptually similar to the analogous result in the framework of nonautonomous difference equations (see Pötzsche \& Rasmussen (2008) and references therein), where complications due to the measurable time-dependence of (2.2) can be treated as in Aulbach \& Wanner (1996). Hence, we present only an outline of the proof. Indeed, direct estimates for the mappings $P^{+}(t) \mathscr{T}_{T}^{ \pm}(t, \phi, \xi)$ and $P^{-}(t) \mathscr{T}_{T}^{ \pm}(t, \phi, \xi)$ yield the Lipschitz estimate (2.10), as well as

$$
\left\|\mathscr{T}_{T}^{ \pm}(\phi, \xi)-\mathscr{T}_{T}^{ \pm}(\phi, \bar{\xi})\right\|_{\tau, \gamma}^{ \pm} \leqslant K_{ \pm}\|\xi-\bar{\xi}\| .
$$

Thanks to (2.9) and (2.10), $\mathscr{T}_{T}^{ \pm}(\cdot, \xi)$ is a contraction on $\mathscr{X}_{\tau, \gamma}^{ \pm}(T)$ and Banach's theorem yields a unique fixed point $\phi_{T}^{ \pm}(\xi) \in \mathscr{X}_{\tau, \gamma}^{ \pm}(T)$. Having this available, (2.11) is a direct consequence of (2.12). 
THEOREM 2.3 (PSEUdo-STABlE AND PSEUdo-UnSTABlE INTEGRAL MANIFOldS) Assume Assumption 2.1-2.2 hold with (2.9), and choose $\sigma \in\left(\ell, \frac{1}{2}(\beta-\alpha)\right]$. Then the following holds true:

(a) If $I$ is unbounded above, then the so-called pseudo-stable manifold

$$
S^{+}:=\left\{(\tau, \xi) \in I \times \mathbb{R}^{d}: \varphi(\cdot ; \tau, \xi) \in \mathscr{X}_{\tau, \gamma}^{+}(\infty) \text { for all } \gamma \in[\alpha+\sigma, \beta-\sigma]\right\}
$$

is an integral manifold of (2.2) possessing the representation

$$
S^{+}=\left\{\left(\tau, \xi+s^{+}(\tau, \xi)\right) \in I \times \mathbb{R}^{d}: \tau \in I, \xi \in R\left(P_{+}(\tau)\right)\right\}
$$

with a uniquely determined continuous mapping $s^{+}: I \times \mathbb{R}^{d} \rightarrow \mathbb{R}^{d}$, given by

$$
s^{+}(\tau, \xi)=P_{-}(\tau) \phi_{\infty}^{+}(\tau, \xi) \text { for all } \tau \in I, \xi \in \mathbb{R}^{d}
$$

Furthermore, $s^{+}(\tau, 0) \equiv 0$ on $I$ and $s^{+}$satisfies $\operatorname{Lip}_{2} s^{+} \leqslant \frac{K_{-} K_{+} L}{\sigma-\ell}$.

(b) If $I$ is unbounded below, then the so-called pseudo-unstable manifold

$$
S^{-}:=\left\{(\tau, \xi) \in I \times \mathbb{R}^{d}: \varphi(\cdot ; \tau, \xi) \in \mathscr{X}_{\tau, \gamma}^{-}(\infty) \text { for all } \gamma \in[\alpha+\sigma, \beta-\sigma]\right\}
$$

is an integral manifold of (2.2) possessing the representation

$$
S^{-}=\left\{\left(\tau, \eta+s^{-}(\tau, \eta)\right) \in I \times \mathbb{R}^{d}: \tau \in I, \eta \in R\left(P_{-}(\tau)\right)\right\}
$$

with a uniquely determined continuous mapping $s^{-}: I \times \mathbb{R}^{d} \rightarrow \mathbb{R}^{d}$, given by

$$
s^{-}(\tau, \eta)=P_{+}(\tau) \phi_{\infty}^{-}(\tau, \eta) \text { for all } \tau \in I, \eta \in \mathbb{R}^{d}
$$

Furthermore, $s^{-}(\tau, 0) \equiv 0$ on $I$ and $s^{-}$satisfies $\operatorname{Lip}_{2} s^{-} \leqslant \frac{K_{-} K_{+} L}{\sigma-\ell}$.

Proof. Proceed as in (Aulbach \& Wanner, 1996, Theorem 4.1).

REMARK 2.2 (1) We relate Theorem 2.3 to the standard autonomous situation, where it yields the classical hierarchy of invariant manifolds: $S^{+}$is of center-stable type in case $\beta>0$, of stable type in the hyperbolic situation $\alpha<0<\beta$ and of strongly stable type for $\beta<0$. In a dual fashion, $S^{-}$is of center-unstable type for $\alpha<0$, of unstable type in the hyperbolic situation $\alpha<0<\beta$ and of strongly unstable type in case $0<\alpha$. Finally, the intersection of center-stable and -unstable manifold yields the center manifold. All these nonautonomous sets allow dynamical interpretations as in the discrete situation of difference equations (cf. Pötzsche \& Rasmussen (2008)).

(2) The global condition (2.9) is hardly met in applications, but for later reference we describe the well-known procedure to circumvent this problem: Indeed, Theorem 2.3 is applicable to differential equations (2.2) with an appropriately modified nonlinearity. More detailed, for functions $F$ satisfying

$$
\lim _{x, \bar{x} \rightarrow 0} \frac{F(t, x)-F(t, \bar{x})}{\|x-\bar{x}\|}=0 \quad \text { uniformly in } t \in I
$$


one defines $F_{\rho}(t, x)=F\left(t, r_{\rho}(x)\right)$ with a radial retraction mapping $r_{\rho}: \mathbb{R}^{d} \rightarrow \mathbb{R}^{d}, r_{\rho}(x)=x$ for $\|x\| \leqslant \rho$ and $r_{\rho}(x)=\frac{x}{\|x\|}$ for $\|x\|>\rho$. Then $F(t, \cdot)$ and $F_{\rho}(t, \cdot)$ coincide on a ball of radius $\rho>0$, it is possible to choose $\rho>0$ so small that $F_{\rho}$ satisfies (2.9) and we can apply our theory to

$$
\dot{x}=A(t) x+F_{\rho}(t, x) .
$$

Inside this ball, (2.2) and (2.15) coincide, share the same dynamics, and the global invariant manifolds $S^{+}, S^{-}$of (2.15) restricted to $\left\{(\tau, \xi) \in I \times \mathbb{R}^{d}:\|\xi\| \leqslant \rho\right\}$ are locally invariant w.r.t. (2.2).

(3) Nonautonomous differential equations of the form (2.2) typically occur as equations of perturbed motion related to (pseudo-) hyperbolic solutions. More detailed, suppose a Carathéodory differential equation $\dot{x}=f(t, x)$, where the right-hand side $f$ is $C^{1}$-smooth in the second variable, has a (pseudo-) hyperbolic complete solution $\phi^{*}: \mathbb{R} \rightarrow \mathbb{R}^{d}$ in the sense that $\dot{x}=D_{1} f\left(t, \phi^{*}(t)\right) x$ admits an exponential dichotomy as in Assumption 2.1. Then Theorem 2.3 can be applied to

$$
\dot{x}=f\left(t, x+\phi^{*}(t)\right)-f\left(t, \phi^{*}(t)\right)
$$

and the integral manifolds $S^{-}, S^{+}$describe the saddle-point structure corresponding to $\phi^{*}$. Numerical algorithms to compute hyperbolic solutions $\phi^{*}$ have been developed in Ju et al. (2003).

\section{Truncated Lyapunov-Perron equations and their solution}

We prove an error estimate for the solutions of a truncated Lyapunov-Perron equation. This reduces our problem of finding a fixed point for $\mathscr{T}_{T}^{ \pm}(\cdot, \xi)$ to a Fredholm integral equation of second kind.

Proposition 3.1 Let $\tau \in I, \xi \in \mathbb{R}^{d}, T>0$, suppose Assumption 2.1-2.2 hold with (2.9) and choose $\sigma \in\left(\ell, \frac{1}{2}(\beta-\alpha)\right)$. Then the function $s^{ \pm}: I \times \mathbb{R}^{d} \rightarrow \mathbb{R}^{d}$ defining the integral manifold $S^{ \pm}$satisfies

$$
\left\|s^{ \pm}(\tau, \xi)-P_{\mp}(\tau) \phi_{T}^{ \pm}(\tau, \xi)\right\| \leqslant \frac{\sigma K_{\mp}^{2} K_{ \pm} L}{(\sigma-\ell)^{2}}\left\|P_{ \pm}(\tau) \xi\right\| e^{(2 \sigma-(\beta-\alpha)) T} .
$$

REMARK 3.1 (SPECTRAL GAP CONDITION) By the choice of $\sigma$, we achieve a good approximation in (3.1) for small values of $T>0$, if the difference $\beta-\alpha$ is large compared to $L$. In the autonomous situation this means that real parts of the spectrum admit large gaps.

Proof. Due to analogy, we only prove the assertion for $s^{-}$and $\phi_{T}^{-}$. Choose a finite positive integer $T$, $\gamma \in(\alpha+\sigma, \beta-\sigma]$, and thanks to $\sigma<\frac{1}{2}(\beta-\alpha)$, we can select a $\delta \in[\alpha+\sigma, \gamma)$. Let $\tau \in I, \xi \in \mathbb{R}^{d}$ be fixed and $\phi_{T}^{-} \in \mathscr{X}_{\tau, \gamma}^{-}(T), \phi_{\infty}^{-} \in \mathscr{X}_{\tau, \gamma}^{-}(\infty)$ be the unique fixed points of the Lyapunov-Perron operator as defined in (2.6). Here we have suppressed the dependence on $\xi$. On the finite interval $[\tau-T, \tau]$, one clearly has $\phi_{T}^{-},\left.\phi_{\infty}^{-}\right|_{\tau_{\tau}^{-}(T)} \in \mathscr{X}_{\tau, \delta}^{-}(T)$, and we deduce from Proposition 2.2 that

$$
\begin{aligned}
& \left\|P_{+}(t)\left[\phi_{\infty}^{-}(t)-\phi_{T}^{-}(t)\right]\right\| e^{\delta(\tau-t)} \stackrel{(2.6)}{\leqslant}\left\|\int_{-\infty}^{\tau-T} \Phi(t, s) P_{+}(s) F\left(s, \phi_{\infty}^{-}(s)\right) \mathrm{d} s\right\| e^{\delta(\tau-t)} \\
& +\left\|\int_{\tau-T}^{t} \Phi(t, s) P_{+}(s)\left[F\left(s, \phi_{\infty}^{-}(s)\right)-F\left(s, \phi_{T}^{-}(s)\right)\right] \mathrm{d} s\right\| e^{\delta(\tau-t)} \\
\stackrel{(2.9)}{\leqslant} & K_{+} L e^{\delta(\tau-t)} \int_{-\infty}^{\tau-T} e^{\alpha(t-s)}\left\|\phi_{\infty}^{-}(s)\right\| \mathrm{d} s+K_{+} L e^{\delta(\tau-t)} \int_{\tau-T}^{t} e^{\alpha(t-s)}\left\|\phi_{\infty}^{-}(s)-\phi_{T}^{-}(s)\right\| \mathrm{d} s \\
\leqslant & \frac{K_{+} L}{\gamma-\alpha} e^{(\delta-\gamma) T}\left\|\phi_{\infty}^{-}\right\|_{\tau, \gamma}^{-}+\frac{K_{+} L}{\delta-\alpha}\left\|\phi_{\infty}^{-}-\phi_{T}^{-}\right\|_{\tau, \delta}^{-} \quad \text { for all } t \in[\tau-T, \tau],
\end{aligned}
$$


and analogously we obtain

$$
\left\|P_{-}(t)\left[\phi_{\infty}^{-}(t)-\phi_{T}^{-}(t)\right]\right\| e^{\delta(\tau-t) \stackrel{(2.6)}{\leqslant}} \frac{K_{-} L}{\beta-\delta}\left\|\phi_{\infty}^{-}-\phi_{T}^{-}\right\|_{\tau, \delta}^{-} \quad \text { for all } t \in[\tau-T, \tau] .
$$

Referring to the definition of the $\|\cdot\|_{\tau, \delta}^{-}$-norm and due to $\gamma, \delta \in[\alpha+\sigma, \beta-\sigma]$, we arrive at

$$
\left\|\phi_{T}^{-}-\phi_{\infty}^{-}\right\|_{\tau, \delta}^{-} \leqslant \frac{K_{+} L}{\sigma} e^{(\delta-\gamma) T}\left\|\phi_{\infty}^{-}\right\|_{\tau, \gamma}^{-}+\frac{\ell}{\sigma}\left\|\phi_{T}^{-}-\phi_{\infty}^{-}\right\|_{\tau, \delta}^{-}
$$

and consequently (note the inequality $\ell<\sigma$ ),

$$
\left\|P_{+}(t)\left[\phi_{\infty}^{-}(t)-\phi_{T}^{-}(t)\right]\right\| e^{\delta(\tau-t)} \leqslant \frac{K_{+}^{2} L}{\sigma-\ell} e^{(\delta-\gamma) T}\left\|\phi_{\infty}^{-}\right\|_{\tau, \gamma}^{-} \quad \text { for all } t \in[\tau-T, \tau] .
$$

Therefore, the claim follows from inequality (2.11) in Proposition 2.2, if we use (2.14) and set $t=\tau$, $\delta=\alpha+\sigma, \gamma=\beta-\sigma$ in the above estimate.

With a subset $I \subseteq \mathbb{R}$ and the function space

$$
\mathscr{P} \mathscr{C}\left(I, \mathbb{R}^{d}\right):=\left\{\begin{array}{l|l}
\phi: I \rightarrow \mathbb{R}^{d} & \begin{array}{l}
\phi \text { is piecewise constant with a } \\
\text { finite number of discontinuities }
\end{array}
\end{array}\right\}
$$

our approximations of $\mathscr{X}_{\tau, \gamma}^{ \pm}(T), T \in(0, \infty]$, are given by the spaces

$$
\tilde{\mathscr{X}}_{\tau, \gamma}^{ \pm}(T):=\left\{\phi \in \mathscr{P} \mathscr{C}\left(I_{\tau}^{ \pm}(T), \mathbb{R}^{d}\right): \sup _{t \in I_{\tau}^{ \pm}(T)} e^{\gamma(\tau-t)}\|\phi(t)\|<\infty\right\}
$$

equipped with the norms (2.4). Note that the condition $\sup _{t \in I_{\tau}^{ \pm}(T)} e^{\gamma(\tau-t)}\|\phi(t)\|<\infty$ is always fulfilled for finite values of $T$, and also that for $\gamma<0$, the elements of the space $\tilde{\mathscr{X}}_{\tau, \gamma}^{+}(\infty)$ are eventually zero for large positive times; the same holds in case of functions in $\tilde{\mathscr{X}}_{\tau, \gamma}^{-}(\infty)$ for large negative times and $\gamma>0$.

In our following step, we try to compute fixed points of the Lyapunov-Perron operators (2.5) and (2.6) for a finite $T>0$. These problems are nonlinear Fredholm integral equations of second kind with Green's function $G(t, s)$ as kernel. Although it is an interesting matter to apply numerical solvers for Fredholm equations directly (cf. Atkinson (1976)), we undertake an ad hoc approach and suggest an iterative scheme. Thereto, some preparations are necessary. First of all, we need an additional

Assumption 3.1 Suppose that the function $A \in \mathscr{L}_{\text {loc }}^{1}\left(I, \mathbb{R}^{d \times d}\right)$ is essentially bounded, i.e., one has

$$
a:=\operatorname{ess} \sup _{t \in I}\|A(t)\|<\infty .
$$

REMARK 3.2 The above boundedness assumption on $A$ implies that $\Phi(t, s)$ satisfies the relations

$$
\|\Phi(t, s)\| \leqslant e^{a|t-s|}, \quad \quad\|\Phi(t, s)-\mathrm{id}\| \leqslant a|t-s| e^{a|t-s|} \quad \text { for all } s, t \in I .
$$

LEMma 3.1 Suppose Assumptions 2.1, 2.2, 3.1 hold with (2.9), $T \in(0, \infty]$ and $\gamma \in[\alpha+\sigma, \beta-\sigma]$. Then, for each $\tau \in I$ and $\xi \in \mathbb{R}^{d}$, one has: 
10 of 30

(a) The fixed point $\phi_{T}^{+}(\xi) \in \mathscr{X}_{\tau, \gamma}^{+}(T)$ of the Lyapunov-Perron operator (2.5) satisfies

$$
\left\|\phi_{T}^{+}(t, \xi)-\phi_{T}^{+}(s, \xi)\right\| \leqslant a(t-s) e^{a(t-s)}\left(K_{+} e^{\alpha(t-s)}\left\|P_{+}(\tau) \xi\right\|+\frac{1+\sigma}{\sigma} \ell\left\|\phi_{T}^{+}(\xi)\right\|_{\tau, \gamma}^{+}\right) e^{\gamma(t-\tau)}
$$

for all $\tau \leqslant s \leqslant t$,

(b) the fixed point $\phi_{T}^{-}(\xi) \in \mathscr{X}_{\tau, \gamma}^{-}(T)$ of the Lyapunov-Perron operator (2.6) satisfies

$$
\left\|\phi_{T}^{-}(t, \xi)-\phi_{T}^{-}(s, \xi)\right\| \leqslant a(s-t) e^{a(s-t)}\left(K_{-} e^{\beta(t-s)}\left\|P_{-}(\tau) \xi\right\|+\frac{1+\sigma}{\sigma} \ell\left\|\phi_{T}^{-}(\xi)\right\|_{\tau, \gamma}^{-}\right) e^{\gamma(t-\tau)}
$$

for all $t \leqslant s \leqslant \tau$.

Proof. Let $\tau \in I, \xi \in \mathbb{R}^{d}$ be fixed and $T \in(0, \infty]$.

(a) Let $\phi_{T}^{+} \in \mathscr{X}_{\tau, \gamma}^{+}(T)$ be the unique fixed point of $\mathscr{T}_{T}^{+}(\cdot, \xi)$, where we omit the dependence on $\xi$. For $\tau \leqslant s \leqslant t$, we decompose the difference $\phi_{T}^{+}(t)-\phi_{T}^{+}(s)$ as follows:

$$
\left\|\phi_{T}^{+}(t)-\phi_{T}^{+}(s)\right\| \leqslant I_{1}\left(I_{2}+I_{3}+I_{4}\right)+I_{5}+I_{6},
$$

with functions $I_{1}:=\|\Phi(t, s)-\mathrm{id}\|, I_{2}:=\left\|\Phi(s, \tau) P_{+}(\tau) \xi\right\|$ and

$$
\begin{array}{rlrl}
I_{3} & :=\left\|\int_{\tau}^{s} \Phi(t, r) P_{+}(r) F\left(r, \phi_{T}^{+}(r)\right) \mathrm{d} r\right\|, & I_{4}:=\left\|\int_{t}^{\tau+T} \Phi(t, r) P_{-}(r) F\left(r, \phi_{T}^{+}(r)\right) \mathrm{d} r\right\|, \\
I_{5}:=\left\|\int_{s}^{t} \Phi(t, r) P_{+}(r) F\left(r, \phi_{T}^{+}(r)\right) \mathrm{d} r\right\|, & I_{6}:=\left\|-\int_{s}^{t} \Phi(s, r) P_{-}(r) F\left(r, \phi_{T}^{+}(r)\right) \mathrm{d} r\right\| .
\end{array}
$$

Using the bounded growth relation (3.2), we immediately obtain $I_{1} \leqslant a(t-s) e^{a(t-s)}$, and by the dichotomy estimates, the Lipschitz condition for $F$, and the relation $\left\|\phi_{T}^{+}(t)\right\| \leqslant e^{\gamma(t-\tau)}\left\|\phi_{T}^{+}\right\|_{\tau, \gamma}^{+}$, we get

$$
\begin{array}{ll}
I_{2} \leqslant K_{+} e^{\alpha(s-t)}\left\|P_{+}(\tau) \xi\right\| e^{\gamma(t-\tau)}, & \\
I_{3} \leqslant \frac{K_{+} L}{\gamma-\alpha}\left\|\phi_{T}^{+}\right\|_{\tau, \gamma}^{+} e^{\gamma(t-\tau)}, & I_{4} \leqslant \frac{K_{-} L}{\beta-\gamma}\left\|\phi_{T}^{+}\right\|_{\tau, \gamma}^{+} e^{\gamma(t-\tau)}, \\
I_{5} \leqslant K_{+} L(t-s)\left\|\phi_{T}^{+}\right\|_{\tau, \gamma}^{+} e^{\gamma(t-\tau)}, & I_{6} \leqslant K_{-} L(t-s)\left\|\phi_{T}^{+}\right\|_{\tau, \gamma}^{+} e^{\gamma(t-\tau)} .
\end{array}
$$

Then the claim follows by assembling these estimates into (3.3).

(b) Referring to (a), we omit the proof of assertion (b) due to duality reasons.

In order to transform (2.5) and (2.6) into a finite-dimensional problem, we introduce a discrete analogue to the Lyapunov-Perron operators $\mathscr{T}_{T}^{ \pm}$as follows: Consider $N \in \mathbb{N}$, pick a finite $T>0$, and note that $\mathscr{T}_{T}^{ \pm}$can also be defined on the function space $\tilde{\mathscr{X}}_{\tau, \gamma}^{ \pm}(T)$. Then, for $\tau \in I, \xi \in \mathbb{R}^{d}$ and $\phi \in$ $\tilde{\mathscr{X}}_{\tau, \gamma}^{ \pm}(T)$, we define the operator $\tilde{\mathscr{T}}_{T, N}^{ \pm}$by

$$
\tilde{\mathscr{T}}_{T, N}^{ \pm}(t, \phi, \xi):=\mathscr{T}_{T}^{ \pm}\left(k_{N}^{ \pm}(t), \phi, \xi\right) \quad \text { for all } t \in I_{\tau}^{ \pm}(T)
$$

where

$$
k_{N}^{+}(t):=\tau+\frac{T}{N}\left\lfloor\frac{(t-\tau) N}{T}\right\rfloor, \quad \quad k_{N}^{-}(t):=\tau+\frac{T}{N}\left\lceil\frac{(t-\tau) N}{T}\right\rceil
$$


Before stating the next result, we introduce the abbreviations

$$
\chi_{\gamma}^{+}(h):=\sup _{0 \leqslant x \leqslant h} e^{\gamma x}=\max \left\{1, e^{\gamma h}\right\}, \quad \chi_{\gamma}^{-}(h):=\sup _{-h \leqslant x \leqslant 0} e^{\gamma x}=\max \left\{1, e^{-\gamma h}\right\}
$$

for reals $h \geqslant 0, \gamma$, observe $\lim _{h \backslash 0} \chi_{\gamma}^{ \pm}(h)=1$ and obtain the following

Lemma 3.2 We suppose that Assumptions 2.1, 2.2 and 3.1 hold with (2.9), $T>0, N \in \mathbb{N}$ and $\gamma \in$ $[\alpha+\sigma, \beta-\sigma]$. Then, for each $\tau \in I, \xi \in \mathbb{R}^{d}$, one has:

(a) The mapping $\tilde{\mathscr{T}}_{T, N}^{+}: \tilde{\mathscr{X}}_{\tau, \gamma}^{+}(T) \times \mathbb{R}^{d} \rightarrow \tilde{\mathscr{X}}_{\tau, \gamma}^{+}(T)$ is well-defined and satisfies

$$
\begin{aligned}
& \left\|\phi_{T}^{+}(\xi)-\tilde{\mathscr{T}}_{T, N}^{+}(\phi, \xi)\right\|_{\tau, \gamma}^{+} \leqslant \frac{\ell}{\sigma} \chi_{-\gamma}^{+}\left(\frac{T}{N}\right)\left\|\phi_{T}^{+}(\xi)-\phi\right\|_{\tau, \gamma}^{+} \\
& \quad+a \frac{T}{N} \chi_{a}^{+}\left(\frac{T}{N}\right)\left(K_{+} \chi_{\alpha}^{+}\left(\frac{T}{N}\right)+\ell \frac{1+\sigma}{\sigma-\ell}\right)\left\|P_{+}(\tau) \xi\right\| \quad \text { for all } \phi \in \tilde{\mathscr{X}}_{\tau, \gamma}^{+}(T),
\end{aligned}
$$

(b) the mapping $\tilde{\mathscr{T}}_{T, N}^{-}: \tilde{\mathscr{X}}_{\tau, \gamma}^{-}(T) \times \mathbb{R}^{d} \rightarrow \tilde{\mathscr{X}}_{\tau, \gamma}^{-}(T)$ is well-defined and satisfies

$$
\begin{aligned}
& \left\|\phi_{T}^{-}(\xi)-\tilde{\mathscr{T}}_{T, N}^{-}(\phi, \xi)\right\|_{\tau, \gamma}^{-} \leqslant \frac{\ell}{\sigma} \chi_{-\gamma}^{-}\left(\frac{T}{N}\right)\left\|\phi_{T}^{-}(\xi)-\phi\right\|_{\tau, \gamma}^{-} \\
& \quad+a \frac{T}{N} \chi_{a}^{+}\left(\frac{T}{N}\right)\left(K_{-} \chi_{\beta}^{-}\left(\frac{T}{N}\right)+\ell \frac{1+\sigma}{\sigma-\ell}\right)\left\|P_{-}(\tau) \xi\right\| \quad \text { for all } \phi \in \tilde{\mathscr{X}}_{\tau, \gamma}^{-}(T)
\end{aligned}
$$

Proof. Let $\tau \in I, \xi \in \mathbb{R}^{d}, \gamma \in[\alpha+\sigma, \beta-\sigma]$ and $T>0$ be finite and fixed.

(a) Choose $t \in[\tau, \tau+T], \phi \in \tilde{\mathscr{X}}_{\tau, \gamma}^{+}(T)$, and let $\phi_{T}^{+} \in \mathscr{X}_{\tau, \gamma}^{+}(T)$ be the unique fixed point of $\mathscr{T}_{T}^{+}(\cdot, \xi)$; we suppress the dependence on $\xi$, and the inclusion $\tilde{\mathscr{T}}_{T, N}^{+}(\phi, \xi) \in \tilde{\mathscr{X}}_{\tau, \gamma}^{+}(T)=\mathscr{P} \mathscr{C}\left(I_{\tau}^{+}(T), \mathbb{R}^{d}\right)$ follows directly. Then we have by definition of $\tilde{\mathscr{T}}_{T, N}^{+}$that

$$
\left\|\phi_{T}^{+}(t)-\tilde{\mathscr{T}}_{T, N}^{+}(t, \phi, \xi)\right\| \leqslant\left\|\phi_{T}^{+}(t)-\phi_{T}^{+}\left(k_{N}^{+}(t)\right)\right\|+\left\|\phi_{T}^{+}\left(k_{N}^{+}(t)\right)-\mathscr{T}_{T}^{+}\left(k_{N}^{+}(t), \phi, \xi\right)\right\| .
$$

For the first summand, we notice that $0 \leqslant t-k_{N}^{+}(t) \leqslant \frac{T}{N}$ and apply Lemma 3.1(a) with $s=k_{N}^{+}(t)$ to obtain

$$
\begin{aligned}
e^{\gamma(\tau-t)}\left\|\phi_{T}^{+}(t)-\phi_{T}^{+}\left(k_{N}^{+}(t)\right)\right\| & \leqslant a \frac{T}{N} \chi_{a}^{+}\left(\frac{T}{N}\right)\left(K_{+} \chi_{\alpha}^{+}\left(\frac{T}{N}\right)\left\|P_{+}(\tau) \xi\right\|+\ell \frac{1+\sigma}{\sigma}\left\|\phi_{T}^{+}(\xi)\right\|_{\tau, \gamma}^{+}\right) \\
& \stackrel{(2.11)}{\leqslant} K_{+} a \frac{T}{N} \chi_{a}^{+}\left(\frac{T}{N}\right)\left(\chi_{\alpha}^{+}\left(\frac{T}{N}\right)+\ell \frac{1+\sigma}{\ell-\sigma}\right)\left\|P_{+}(\tau) \xi\right\| .
\end{aligned}
$$

In second instance, we consider the second term in the above estimate and readily observe the identity $\phi_{T}^{+}\left(k_{N}^{+}(t)\right)=\mathscr{T}_{T}^{+}\left(k_{N}^{+}(t), \phi_{T}^{+}, \xi\right)$. Thanks to $0 \leqslant t-k_{N}^{+}(t) \leqslant \frac{T}{N}$, this implies that

$$
\begin{aligned}
e^{\gamma(\tau-t)}\left\|\phi_{T}^{+}\left(k_{N}^{+}(t)\right)-\mathscr{T}_{T}^{+}\left(k_{N}^{+}(t), \phi, \xi\right)\right\| & \leqslant \chi_{-\gamma}^{+}\left(\frac{T}{N}\right) e^{\gamma\left(\tau-k_{N}^{+}(t)\right)}\left\|\phi_{T}^{+}\left(k_{N}^{+}(t)\right)-\mathscr{T}_{T}^{+}\left(k_{N}^{+}(t), \phi, \xi\right)\right\| \\
& \leqslant \chi_{-\gamma}^{+}\left(\frac{T}{N}\right)\left\|\phi_{T}^{+}-\mathscr{T}_{T}^{+}(\phi, \xi)\right\|_{\tau, \gamma}^{+} \stackrel{(2.10)}{\leqslant} \chi_{-\gamma}^{+}\left(\frac{T}{N}\right) \frac{\ell}{\sigma}\left\|\phi_{T}^{+}-\phi\right\|_{\tau, \gamma}^{+} .
\end{aligned}
$$


Hence, we obtain

$$
\begin{aligned}
e^{\gamma(\tau-t)}\left\|\phi_{T}^{+}(t)-\tilde{\mathscr{T}}_{T, N}^{+}(t, \phi, \xi)\right\| \leqslant & \chi_{-\gamma}^{+}\left(\frac{T}{N}\right) \frac{\ell}{\sigma}\left\|\phi_{T}^{+}-\phi\right\|_{\tau, \gamma}^{+} \\
& +K_{+} a \frac{T}{N} \chi_{a}^{+}\left(\frac{T}{N}\right)\left(\chi_{\alpha}^{+}\left(\frac{T}{N}\right)+\ell \frac{1+\sigma}{\ell-\sigma}\right)\left\|P_{+}(\tau) \xi\right\| \quad \text { for all } \tau \leqslant t,
\end{aligned}
$$

and this yields the desired estimate.

(b) Using Lemma 3.1(b) and Proposition 2.2, this is shown analogously to (a).

Now let $\left(N_{n}\right)_{n \in \mathbb{N}}$ be a strictly increasing sequence of positive integers and consider the recursion

$$
\psi_{n}^{ \pm}:=\tilde{\mathscr{T}}_{T, N_{n}}^{ \pm}\left(\psi_{n-1}^{ \pm}, \xi\right) \quad \text { for all } n \in \mathbb{N}
$$

with constant initial function $\psi_{0}^{ \pm}(t) \equiv P_{ \pm}(\tau) \xi$ satisfying $\psi_{0}^{ \pm} \in \mathscr{X}_{\tau, \gamma}^{ \pm}(T)$. Convergence of the sequence $\left(\psi_{n}^{ \pm}\right)_{n \in \mathbb{N}_{0}}$ will be shown now.

THEOREM 3.2 Suppose that Assumptions 2.1, 2.2, 3.1 hold with (2.9), assume $T>0$ and choose a decay rate $q \in\left(\frac{\ell}{\sigma}, 1\right)$. If the sequence $\left(N_{n}\right)_{n \in \mathbb{N}}$ is as above with

$$
c q^{-n} \leqslant N_{n} \quad \text { for all } n \in \mathbb{N}, \quad \quad \ell \chi_{-\gamma}^{ \pm}\left(\frac{T}{N_{1}}\right) \leqslant \sigma q, \quad \chi_{a}^{+}\left(\frac{T}{N_{1}}\right) \leqslant 2
$$

for some $c>0$, then for all $\tau \in I, \xi \in \mathbb{R}^{d}$, the following holds:

(a) Under the assumption $\chi_{\alpha}^{+}\left(\frac{T}{N_{1}}\right) \leqslant 2$, one has

$$
\left\|\phi_{T}^{+}(\tau, \xi)-\psi_{n}^{+}(\tau)\right\| \leqslant\left(C_{1}^{+}+C_{2}^{+} n\right) q^{n}\left\|P_{+}(\tau) \xi\right\| \quad \text { for all } n \in \mathbb{N}
$$

(b) and under the assumption $\chi_{\beta}^{-}\left(\frac{T}{N_{1}}\right) \leqslant 2$, one has

$$
\left\|\phi_{T}^{-}(\tau, \xi)-\psi_{n}^{-}(\tau)\right\| \leqslant\left(C_{1}^{-}+C_{2}^{-} n\right) q^{n}\left\|P_{-}(\tau) \xi\right\| \quad \text { for all } n \in \mathbb{N},
$$

where $C_{1}^{ \pm}, C_{2}^{ \pm}>0$ are given by

$$
C_{1}^{ \pm}:=\chi_{\gamma}^{+}(T)+\frac{K_{ \pm} L}{\sigma-\ell}, \quad C_{2}^{ \pm}:=2 a c T\left(2 K_{ \pm}+\ell \frac{1+\sigma}{\sigma-\ell}\right) .
$$

Proof. Let $\tau \in I, \xi \in \mathbb{R}^{d}$ and $\gamma \in[\alpha+\sigma, \beta-\sigma]$.

(a) Using mathematical induction, one gets from Lemma 3.2(a) that, provided $\chi_{\alpha}^{+}\left(T / N_{1}\right) \leqslant 2$, the sequence $\left(\psi_{n}^{+}\right)_{n \in \mathbb{N}}$ in $\tilde{\mathscr{X}}_{\tau, \gamma}^{+}(T)$ satisfies the estimate

$$
\left\|\phi_{T}^{+}(\xi)-\psi_{n}^{+}\right\|_{\tau, \gamma}^{+} \leqslant q^{n}\left\|\phi_{T}^{+}(\xi)-\psi_{0}^{+}\right\|_{\tau, \gamma}^{+}+2 a T\left(2 K_{+}+\ell \frac{1+\sigma}{\sigma-\ell}\right)\left\|P_{+}(\tau) \xi\right\| \sum_{j=0}^{n-1} \frac{q^{j}}{N_{n-j}} \quad \text { for all } n \in \mathbb{N} \text {. }
$$

Then, our choice of the initial condition $\psi_{0}^{+}$implies $\left\|\psi_{0}^{+}\right\|_{\tau, \gamma}^{+} \leqslant \chi_{\gamma}^{+}(T)\left\|P_{+}(\tau) \xi\right\|$, and due to Proposition 2.2, we obtain from the triangle inequality that

$$
\begin{aligned}
\left\|\phi_{T}^{+}(\xi)-\psi_{n}^{+}\right\|_{\tau, \gamma}^{+} \stackrel{(2.11)}{\leqslant}\left(\chi_{\gamma}^{+}(T)+\frac{K_{+} L}{\sigma-\ell}\right) q^{n}\left\|P_{+}(\tau) \xi\right\| & \\
& +2 a T\left(2 K_{+}+\ell \frac{1+\sigma}{\sigma-\ell}\right)\left\|P_{+}(\tau) \xi\right\| \sum_{j=0}^{n-1} \frac{q^{j}}{N_{n-j}} \quad \text { for all } n \in \mathbb{N} .
\end{aligned}
$$


Referring to (3.7), this implies our relation (3.8).

(b) Similarly to (a), one uses Lemma 3.2(b) and mathematical induction to deduce the estimate

$$
\left\|\phi_{T}^{-}(\xi)-\psi_{n}^{-}\right\|_{\tau, \gamma}^{-} \leqslant q^{n}\left\|\phi_{T}^{-}(\xi)-\psi_{0}^{-}\right\|_{\tau, \gamma}^{-}+2 a T\left(2 K_{-}+\ell \frac{1+\sigma}{\sigma-\ell}\right)\left\|P_{-}(\tau) \xi\right\| \sum_{j=0}^{n-1} \frac{q^{j}}{N_{n-j}}
$$

for all $n \in \mathbb{N}$. Then the remaining proof results as in (a) using Proposition 2.2.

Algorithm 3.3 (approximation of $s^{ \pm}$) Choose an accuracy $\varepsilon>0$, initial pairs $\tau \in I, \xi \in R\left(P_{ \pm}(\tau)\right.$ ) and $\sigma \in\left(\ell, \frac{\beta-\alpha}{2}\right)$.

(1) Set $n:=1, \psi_{0}^{ \pm}(t): \equiv \xi$ and a real $T>0$ sufficiently large with

$$
\frac{\sigma K_{\mp}^{2} K_{ \pm} L}{(\sigma-\ell)^{2}}\left\|P_{ \pm}(\tau) \xi\right\| e^{(2 \sigma-(\beta-\alpha)) T}<\frac{\varepsilon}{2}
$$

(2) choose a sequence $\left(N_{n}\right)_{n \in \mathbb{N}}$ according to the assumptions of Theorem 3.2 and that (3.7) holds;

(3) compute $\psi_{n}^{ \pm}:=\tilde{\mathscr{T}}_{T, N_{n}}\left(\psi_{n-1}^{ \pm}, \xi\right)$;

(4) if $\left(C_{1}^{ \pm}+C_{2}^{ \pm} n\right) q^{n}\left\|P_{ \pm}(\tau) \xi\right\| \geqslant \frac{\varepsilon}{2}$, then increase $n$ by 1 and go to (3);

(5) $\operatorname{set} \tilde{s}^{ \pm}(\tau, \xi):=P_{\mp}(\tau) \psi_{n}^{ \pm}(\tau)$.

Thus, the distance between the approximate integral manifold $\tilde{s}^{ \pm}(\tau, \xi)$ and $s^{ \pm}(\tau, \xi)$ satisfies

$$
\left\|s^{ \pm}(\tau, \xi)-\tilde{s}^{ \pm}(\tau, \xi)\right\|<\varepsilon .
$$

\section{Approximation of center-like integral manifolds}

We introduce another method to approximate integral manifolds, which is strongly inspired by the autonomous center-manifold situation considered in Jolly \& Rosa (2005). Thereto, we have to impose a further assumption which seemingly restricts our considerations to center-like manifolds.

ASSUMPTION 4.1 Suppose $\beta>\sigma$ (in case $S^{+}$is concerned) and $\alpha>-\sigma$ (in case $S^{-}$is concerned).

REMARK 4.1 Assumption 4.1 can always be satisfied by applying the so-called spectral transformation $y=e^{\kappa(t-\tau)} x$ to (2.2), which yields the shifted system

$$
\dot{y}=(A(t)+\kappa \cdot \mathrm{id}) y+e^{\kappa(t-\tau)} F\left(t, e^{-\kappa(t-\tau)} y\right),
$$

where $\kappa \in \mathbb{R}$ has to be chosen such that $\beta+\kappa>\sigma$ (in case $S^{+}$is concerned) or $\alpha+\kappa>-\sigma$ (in case $S^{-}$ is concerned), and $\tau$ is used for the approximation of the $\tau$-fiber. One easily verifies that the assumptions of Theorem 2.3 also hold for system (4.1), and this gives rise to invariant pseudo-stable and -unstable manifolds $\tilde{S}^{+}$and $\tilde{S}^{-}$of (4.1), respectively. It is shown in (Aulbach et al., 2006, Theorem 5.1) that we have the relations

$$
\tilde{S}^{+}(t)=e^{\kappa(t-\tau)} S^{+}(t) \quad \text { and } \quad \tilde{S}^{-}(t)=e^{\kappa(t-\tau)} S^{-}(t) \quad \text { for all } t \in I
$$

and this means that the $\tau$-fibers of the manifolds coincide. Hence, the $\tau$-fiber of $S^{+}$or $S^{-}$can be approximated by computing the $\tau$-fiber of $\tilde{S}^{+}$or $\tilde{S}^{+}$, respectively, and this is possible with the algorithm 
proposed in this section, since Assumption 4.1 is fulfilled for system (4.1). We like to emphasize that such a transformation is of no use in a purely autonomous context, since the resulting system (4.1) is nonautonomous even if (2.2) is an autonomous system.

In our present set-up, a discretized version of the Lyapunov-Perron operators $\mathscr{T}_{\infty}^{ \pm}$can be constructed as follows: Let us consider $h>0, N \in \mathbb{N}$ and $\gamma \in[\alpha+\sigma, \beta-\sigma]$, and note that the original map $\mathscr{T}_{\infty}^{ \pm}$is also well-defined on $\tilde{\mathscr{X}}_{\tau, \gamma}^{ \pm}(\infty)$. Then, for $\tau \in I, \xi \in \mathbb{R}^{d}$ and $\phi \in \tilde{\mathscr{X}}_{\tau, \gamma}^{ \pm}(\infty)$, we set

$$
\tilde{\mathscr{T}}_{h, N}^{ \pm}(t, \phi, \xi):=\mathscr{T}_{\infty}^{ \pm}\left(k_{N}^{ \pm}(t), \phi, \xi\right) \quad \text { for all } t \in I_{\tau}^{ \pm}(\infty),
$$

where

$$
k_{N}^{+}(t):=\left\{\begin{array}{l}
\tau+h\left\lfloor\frac{t-\tau}{h}\right\rfloor, \quad 0 \leqslant t-\tau<h N \\
\tau+h N, \quad h N \leqslant t-\tau
\end{array} \quad, \quad k_{N}^{-}(t):=\left\{\begin{array}{l}
\tau+h\left\lceil\frac{t-\tau}{h}\right\rceil, \quad-h N<t-\tau \leqslant 0 \\
\tau-h N, \quad t-\tau \leqslant-h N
\end{array} .\right.\right.
$$

Note that the definition of the functions $k_{N}^{ \pm}$differs from the definition used in the previous section.

We start with an elementary, yet useful lemma. It will be applied to the solutions $\phi_{\infty}^{ \pm}(\xi) \in \mathscr{X}_{\tau, \gamma}^{ \pm}(\infty)$ of (2.2) and consequently could be deduced from Lemma 3.1. Nevertheless, for the sake of simpler constants, we give a direct proof.

Lemma 4.1 Let $\gamma \in[\alpha+\sigma, \beta-\sigma]$, and suppose that Assumptions 2.1, 2.2, 3.1, 4.1 hold.

(a) If $\gamma \geqslant 0$, then any solution $\phi \in \mathscr{X}_{\tau, \gamma}^{+}(\infty)$ of (2.2) satisfies

$$
\|\phi(t)-\phi(s)\| \leqslant(a+L)(t-s) e^{\gamma(t-\tau)}\|\phi\|_{\tau, \gamma}^{+} \quad \text { for all } \tau \leqslant s \leqslant t,
$$

(b) if $\gamma \leqslant 0$, then any solution $\phi \in \mathscr{X}_{\tau, \gamma}^{-}(\infty)$ of (2.2) satisfies

$$
\|\phi(t)-\phi(s)\| \leqslant(a+L)(s-t) e^{\gamma(t-\tau)}\|\phi\|_{\tau, \gamma}^{-} \quad \text { for all } t \leqslant s \leqslant \tau .
$$

Proof. Given $\tau, s, t \in I$, we have

$$
\begin{aligned}
\|\phi(t)-\phi(s)\| & =\left\|\int_{s}^{t}(A(r) \phi(r)+F(r, \phi(r))) \mathrm{d} r\right\| \leqslant \int_{s}^{t}(a+L)\|\phi(r)\| \mathrm{d} r \\
& =(a+L) \int_{s}^{t} e^{\gamma(r-\tau)}\|\phi\|_{\tau, \gamma}^{+} \mathrm{d} r \leqslant(a+L)\|\phi\|_{\tau, \gamma}^{+} \int_{s}^{t} e^{\gamma(t-\tau)} \mathrm{d} r \\
& =(a+L)\|\phi\|_{\tau, \gamma}^{+}(t-s) e^{\gamma(t-\tau)} \quad \text { for all } \tau \leqslant s \leqslant t .
\end{aligned}
$$

This implies (a); assertion (b) can be shown similarly.

REMARK 4.2 Note that the integral $\int_{s}^{t} e^{\gamma(r-\tau)} \mathrm{d} r$ in the above proof can be computed explicitly, providing a sharper estimate. For the sake of clarity, however, we use the inequality as stated in the lemma in our following considerations.

Lemma 4.2 Suppose Assumptions 2.1, 2.2, 3.1, 4.1 hold with (2.9) and $h>0, N \in \mathbb{N}, \gamma \in[\alpha+\sigma, \beta-$ $\sigma]$. Then, for each $\tau \in I, \xi \in \mathbb{R}^{d}$, one has: 
(a) If $0 \leqslant \delta<\gamma$, then the mapping $\tilde{\mathscr{T}}_{h, N}^{+}: \tilde{\mathscr{X}}_{\tau, \gamma}^{+}(\infty) \times \mathbb{R}^{d} \rightarrow \tilde{\mathscr{X}}_{\tau, \gamma}^{+}(\infty)$ is well-defined and satisfies

$$
\begin{aligned}
& \left\|\phi_{\infty}^{+}(\xi)-\tilde{\mathscr{T}}_{h, N}^{+}(\phi, \xi)\right\|_{\tau, \gamma}^{+} \leqslant \frac{\ell}{\sigma}\left\|\phi_{\infty}^{+}(\xi)-\phi\right\|_{\tau, \gamma}^{+} \\
& \quad+(a+L) \max \left\{h\left\|\phi_{\infty}^{+}(\xi)\right\|_{\tau, \gamma}^{+}, \frac{e^{(\delta-\gamma) N h-1}}{\gamma-\delta}\left\|\phi_{\infty}^{+}(\xi)\right\|_{\tau, \delta}^{+}\right\} \quad \text { for all } \phi \in \tilde{\mathscr{X}}_{\tau, \gamma}^{+}(\infty),
\end{aligned}
$$

(b) if $\gamma<\delta \leqslant 0$, then the mapping $\tilde{\mathscr{T}}_{h, N}^{-}: \tilde{\mathscr{X}}_{\tau, \gamma}^{-}(\infty) \times \mathbb{R}^{d} \rightarrow \tilde{\mathscr{X}}_{\tau, \gamma}^{-}(\infty)$ is well-defined and satisfies

$$
\begin{aligned}
& \left\|\phi_{\infty}^{-}(\xi)-\tilde{\mathscr{T}}_{h, N}^{-}(\phi, \xi)\right\|_{\tau, \gamma}^{-} \leqslant \frac{\ell}{\sigma}\left\|\phi_{\infty}^{-}(\xi)-\phi\right\|_{\tau, \gamma}^{-} \\
& \quad+(a+L) \max \left\{h\left\|\phi_{\infty}^{-}(\xi)\right\|_{\tau, \gamma}^{-}, \frac{e^{(\gamma-\delta) N h-1}}{\delta-\gamma}\left\|\phi_{\infty}^{+}(\xi)\right\|_{\tau, \delta}^{-}\right\} \quad \text { for all } \phi \in \tilde{\mathscr{X}}_{\tau, \gamma}^{-}(\infty) .
\end{aligned}
$$

Proof. Let $\tau \in I, \xi \in \mathbb{R}^{d}$ and $\gamma \in[\alpha+\sigma, \beta-\sigma]$.

(a) Choose $t \geqslant \tau$ and $\phi \in \tilde{\mathscr{X}}_{\tau, \gamma}^{+}(\infty)$. We clearly have the inclusion $\tilde{\mathscr{T}}_{h, N}^{+}(\phi, \xi) \in \tilde{\mathscr{X}}_{\tau, \gamma}^{+}(\infty)$. Moreover, by definition of $\tilde{\mathscr{T}}_{h, N}^{+}$we get

$$
\left\|\phi_{\infty}^{+}(t)-\tilde{\mathscr{T}}_{h, N}^{+}(t, \phi, \xi)\right\| \leqslant\left\|\phi_{\infty}^{+}(t)-\phi_{\infty}^{+}\left(k_{N}^{+}(t), \xi\right)\right\|+\left\|\phi_{\infty}^{+}\left(k_{N}^{+}(t), \xi\right)-\mathscr{T}_{\infty}^{+}\left(k_{N}^{+}(t), \phi, \xi\right)\right\| .
$$

Proceeding like in the proof of Lemma 3.2(a) we can bound the second term as

$$
e^{\gamma(\tau-t)}\left\|\phi_{\infty}^{+}\left(k_{N}^{+}(t), \xi\right)-\mathscr{T}_{\infty}^{+}\left(k_{N}^{+}(t), \phi, \xi\right)\right\| \leqslant \frac{\ell}{\sigma}\left\|\phi_{\infty}^{+}(\xi)-\phi\right\|_{\tau, \gamma}^{+}
$$

For the first term, we consider the case $t \in[\tau, \tau+N h)$. By construction, the fixed point $\phi_{\infty}^{+}(\xi)$ is a solution of (2.2) in $\mathscr{X}_{\tau, \gamma}^{+}(\infty)$. Thus, we can apply Lemma 4.1(a) with $s=k_{N}^{+}(t)$ and obtain

$$
\begin{aligned}
e^{\gamma(\tau-t)}\left\|\phi_{\infty}^{+}(t)-\phi_{\infty}^{+}\left(k_{N}^{+}(t), \xi\right)\right\| \stackrel{(4.2)}{\leqslant} e^{\gamma(\tau-t)}(a+L)\left(t-k_{N}^{+}(t)\right) e^{\gamma(t-\tau)}\left\|\phi_{\infty}^{+}(\xi)\right\|_{\tau, \gamma}^{+} \\
\leqslant h(a+L)\left\|\phi_{\infty}^{+}(\xi)\right\|_{\tau, \gamma}^{+} .
\end{aligned}
$$

For the remaining situation $t \geqslant \tau+N h$, apply Lemma 4.1(a) with a growth rate $\delta \in[0, \gamma)$ instead of $\gamma$. Thanks to the inclusion $\delta \in[\alpha+\sigma, \beta-\sigma]$, we have $\phi_{\infty}^{+}(\xi) \in \mathscr{X}_{\tau, \delta}^{+}(\infty)$, and the elementary estimate $x e^{(\delta-\gamma) x} \leqslant \frac{e^{-1}}{\gamma-\delta}$ for all $x \geqslant 0$ leads to

$$
\begin{aligned}
e^{\gamma(\tau-t)}\left\|\phi_{\infty}^{+}(t)-\phi_{\infty}^{+}(\tau+N h, \xi)\right\| \stackrel{(4.2)}{\leqslant} e^{\gamma(\tau-t)}(a+L)(t-\tau-N h) e^{\delta(t-\tau)}\left\|\phi_{\infty}^{+}(\xi)\right\|_{\tau, \delta}^{+} \\
=(a+L) e^{(\delta-\gamma) N h}(t-\tau-N h) e^{(\delta-\gamma)(t-\tau-N h)}\left\|\phi_{\infty}^{+}(\xi)\right\|_{\tau, \delta}^{+} \\
\leqslant(a+L) \frac{e^{(\delta-\gamma) N h-1}}{\gamma-\delta}\left\|\phi_{\infty}^{+}(\xi)\right\|_{\tau, \delta}^{+} .
\end{aligned}
$$


From this one immediately obtains the final estimate

$$
\begin{aligned}
e^{\gamma(\tau-t)}\left\|\phi_{\infty}^{+}(t)-\mathscr{T}_{h, N}^{+}(t, \phi, \xi)\right\| & \leqslant \frac{\ell}{\sigma}\left\|\phi_{\infty}^{+}(\xi)-\phi\right\|_{\tau, \gamma}^{+} \\
& +(a+L) \max \left\{h\left\|\phi_{\infty}^{+}(\xi)\right\|_{\tau, \gamma}^{+}, \frac{e^{(\delta-\gamma) N h-1}}{\gamma-\delta}\left\|\phi_{\infty}^{+}(\xi)\right\|_{\tau, \delta}^{+}\right\} \quad \text { for all } \tau \leqslant t,
\end{aligned}
$$

which implies our claim.

(b) Assertion (b) can be shown analogously using Lemma 4.1(b).

For given initial data $\tau \in I$ and $\xi \in \mathbb{R}^{d}$, the above statement concerning the well-definedness of $\mathscr{T}_{h, N}^{ \pm}$allows us to compute the fixed point $\phi_{\infty}^{ \pm}(\xi)$ recursively. Thereto, we prescribe a nonnegative real sequence $\left(h_{n}\right)_{n \in \mathbb{N}}$ and a sequence of nonnegative integers $\left(N_{n}\right)_{n \in \mathbb{N}}$. Then our recursion reads as

$$
\psi_{n}^{ \pm}:=\tilde{\mathscr{T}}_{h_{n}, N_{n}}^{ \pm}\left(\psi_{n-1}^{ \pm}, \xi\right) \text { for all } n \in \mathbb{N},
$$

where we choose the constant initial function $\psi_{0}^{ \pm}(t) \equiv P_{ \pm}(\tau) \xi$. It is quite natural to assume that the sequence $\left(h_{n}\right)_{n \in \mathbb{N}}$ is decreasing such that $\left(N_{n} h_{n}\right)_{n \in \mathbb{N}}$ increases. Due to a compact notation, we define $N_{0}:=0$ and set $h_{0}>0$ arbitrarily.

After all these preparations, we can show the following approximation result.

THEOREM 4.1 Suppose Assumptions 2.1, 2.2, 3.1, 4.1 hold with (2.9). Let the sequence $\left(h_{n}\right)_{n \in \mathbb{N}}$ decrease exponentially and the length of the time intervals $\left(h_{n} N_{n}\right)_{n \in \mathbb{N}}$ increase linearly, i.e., more precisely, assume that there exist reals $c_{1}, c_{2}>0$ such that

$$
0<h_{n} \leqslant c_{1}\left(\frac{\ell}{\sigma}\right)^{n} \quad \text { and } \quad N_{n} h_{n} \geqslant c_{2} n \text { for all } n \in \mathbb{N},
$$

where

$$
\Delta_{+}:=\beta-\sigma-\max \{0, \alpha+\sigma\}, \quad \Delta_{-}:=\min \{0, \beta-\sigma\}-\alpha-\sigma .
$$

Then, for each $\tau \in I, \xi \in \mathbb{R}^{d}$ and all $n \in \mathbb{N}$, it holds

$$
\left\|s^{ \pm}(\tau, \xi)-P_{\mp}(\tau) \psi_{n}^{ \pm}(\tau)\right\| \leqslant K_{\mp}\left(C_{1}^{ \pm}\left(\frac{\ell}{\sigma}\right)^{n}+C_{2}^{ \pm} n q^{n}\right)\left\|P_{ \pm}(\tau) \xi\right\|,
$$

where $q:=\max \left\{\frac{\ell}{\sigma}, e^{-c_{2} \Delta_{ \pm}}\right\} \in(0,1)$ and $C_{1}^{ \pm}, C_{2}^{ \pm}>0$ are given by

$$
C_{1}^{ \pm}:=1+\frac{K_{ \pm} \sigma}{\sigma-\ell}, \quad C_{2}^{ \pm}:=(a+L) \max \left\{c_{1}, \frac{e^{-1}}{\Delta_{ \pm}}\right\} \frac{K_{ \pm} \sigma}{\sigma-\ell} .
$$

Proof. Let $\tau \in I, \xi \in \mathbb{R}^{d}$ and $\gamma \in[\alpha+\sigma, \beta-\sigma]$.

(I) Mathematical induction and Lemma 4.2(a) yields that in case $0 \leqslant \delta<\gamma$, the sequence $\left(\psi_{n}^{+}\right)_{n \in \mathbb{N}}$ in $\tilde{\mathscr{X}}_{\tau, \gamma}^{+}(\infty)$ satisfies the estimate

$$
\begin{aligned}
& \left\|\phi_{\infty}^{+}(\xi)-\psi_{n}^{+}\right\|_{\tau, \gamma}^{+} \leqslant\left(\frac{\ell}{\sigma}\right)^{n}\left\|\phi_{\infty}^{+}(\xi)-\psi_{0}^{+}\right\|_{\tau, \gamma}^{+} \\
& \quad+(a+L) \sum_{j=0}^{n-1}\left(\frac{\ell}{\sigma}\right)^{j} \max \left\{h_{n-j}\left\|\phi_{\infty}^{+}(\xi)\right\|_{\tau, \gamma}^{+}, \frac{e^{(\delta-\gamma) N_{n-j} h_{n-j}-1}}{\gamma-\delta}\left\|\phi_{\infty}^{+}(\xi)\right\|_{\tau, \delta}^{+}\right\} .
\end{aligned}
$$


Since our choice of the initial condition $\psi_{0}^{+}$implies $\left\|\psi_{0}^{+}\right\|_{\tau, \gamma}^{+} \leqslant\left\|P_{+}(\tau) \xi\right\|$, we consequently obtain from Proposition 2.2, and the triangle inequality that

$$
\begin{aligned}
\left\|\phi_{\infty}^{+}(\xi)-\psi_{n}^{+}\right\|_{\tau, \gamma}^{+} \stackrel{(2.11)}{\leqslant}(1 & \left.+\frac{K_{+} \sigma}{\sigma-\ell}\right)\left(\frac{\ell}{\sigma}\right)^{n}\left\|P_{+}(\tau) \xi\right\| \\
& +(a+L) \frac{K_{+} \sigma}{\sigma-\ell}\left\|P_{+}(\tau) \xi\right\| \sum_{j=0}^{n-1}\left(\frac{\ell}{\sigma}\right)^{j} \max \left\{h_{n-j}, \frac{e^{-1-(\gamma-\delta) N_{n-j} h_{n-j}}}{\gamma-\delta}\right\} .
\end{aligned}
$$

We minimize the right hand side by choosing $\gamma:=\beta-\sigma, \delta:=\max \{0, \alpha+\sigma\}$ to arrive at

$$
\begin{aligned}
\left\|\phi_{\infty}^{+}(\xi)-\psi_{n}^{+}\right\|_{\tau, \gamma}^{+} \leqslant\left(1+\frac{K_{+} \sigma}{\sigma-\ell}\right)\left(\frac{\ell}{\sigma}\right)^{n}\left\|P_{+}(\tau) \xi\right\| & \\
& +(a+L) \frac{K_{+} \sigma}{\sigma-\ell}\left\|P_{+}(\tau) \xi\right\| \sum_{j=0}^{n-1}\left(\frac{\ell}{\sigma}\right)^{j} \max \left\{h_{n-j}, \frac{e^{-1-\Delta_{+} N_{n-j} h_{n-j}}}{\Delta_{+}}\right\} .
\end{aligned}
$$

Under the hypotheses for the convergence behavior of the sequences $\left(h_{n}\right)_{n \in \mathbb{N}}$ and $\left(N_{n} h_{n}\right)_{n \in \mathbb{N}}$, one obtains uniform convergence for bounded values of $\xi$. More precisely, we have

$$
\left\|\phi_{\infty}^{+}(\xi)-\psi_{n}^{+}\right\|_{\tau, \gamma}^{+} \leqslant\left(1+\frac{K_{+} \sigma}{\sigma-\ell}\right)\left(\frac{\ell}{\sigma}\right)^{n}\left\|P_{+}(\tau) \xi\right\|+(a+L) \frac{K_{+} \sigma}{\sigma-\ell} C_{3} n q^{n}\left\|P_{+}(\tau) \xi\right\| \quad \text { for all } n \in \mathbb{N}
$$

with the constant $C_{3}:=\max \left\{c_{1}, \frac{e^{-1}}{\Delta_{+}}\right\}$and the assertion follows, since we have

$$
\left\|s^{+}(\tau, \xi)-P_{-}(\tau) \psi_{n}^{+}(\tau)\right\| \stackrel{(2.13)}{\leqslant} K_{-}\left\|\phi_{\infty}^{+}(\tau, \xi)-\psi_{n}^{+}(\tau)\right\| \leqslant\left\|\phi_{\infty}^{+}(\xi)-\psi_{n}^{+}\right\|_{\tau, \gamma}^{+} \quad \text { for all } n \in \mathbb{N}
$$

(II) In case $\gamma<\delta \leqslant 0$, we deduce from Lemma 4.2(b) that the sequence $\left(\psi_{n}^{-}\right)_{n \in \mathbb{N}}$ in $\tilde{\mathscr{X}}_{\tau, \gamma}^{-}(\infty)$ satisfies

$$
\begin{aligned}
& \left\|\phi_{\infty}^{-}(\xi)-\psi_{n}^{-}\right\|_{\tau, \gamma}^{-} \leqslant\left(\frac{\ell}{\sigma}\right)^{n}\left\|\phi_{\infty}^{-}(\xi)-\psi_{0}^{-}\right\|_{\tau, \gamma}^{-} \\
& \quad+(a+L) \sum_{j=0}^{n-1}\left(\frac{\ell}{\sigma}\right)^{j} \max \left\{h_{n-j}\left\|\phi_{\infty}^{-}(\xi)\right\|_{\tau, \gamma}^{-}, \frac{e^{(\gamma-\delta) N_{n-j} h_{n-j}-1}}{\delta-\gamma}\left\|\phi_{\infty}^{-}(\xi)\right\|_{\tau, \delta}^{-}\right\}
\end{aligned}
$$

for all $n \in \mathbb{N}$. Then the proof for $s^{-}$follows analogously to (I) by Proposition 2.2.

Algorithm 4.2 (approximation of $s^{ \pm}$, center-like case) Choose an accuracy $\varepsilon>0$, initial pairs $\tau \in I$, $\xi \in R\left(P_{ \pm}(\tau)\right), \sigma \in\left(\ell, \frac{1}{2}(\beta-\alpha)\right)$ and sequences $\left(h_{n}\right)_{n \in \mathbb{N}},\left(N_{n}\right)_{n \in \mathbb{N}}$ according to (4.5).

(1) Set $n:=1, \psi_{0}^{ \pm}(t): \equiv \xi$;

(2) compute $\psi_{n}^{ \pm}:=\tilde{\mathscr{T}}_{h_{n}, N_{n}}^{ \pm}\left(\psi_{n-1}^{ \pm}, \xi\right)$;

(3) if $K_{\mp}\left(C_{1}^{ \pm}\left(\frac{\ell}{\sigma}\right)^{n}+C_{2}^{ \pm} n q^{n}\right)\left\|P_{ \pm}(\tau) \xi\right\| \geqslant \varepsilon$, then increase $n$ by 1 and go to (2);

(4) $\operatorname{set} \tilde{s}^{ \pm}(\tau, \xi):=P_{\mp}(\tau) \psi_{n}^{ \pm}(\tau)$.

Then the distance between the approximate integral manifold $\tilde{s}^{ \pm}(\tau, \xi)$ and $s^{ \pm}(\tau, \xi)$ fulfills (3.10). 


\section{Implementation, examples and illustrations}

Our overall approach is based on the premise that the transition matrix $\Phi(t, s) \in \mathbb{R}^{d \times d}$ of (2.1) is available. In practice, it has to be approximated using ODE solvers applied to equation (2.1), whereat higher order numerical schemes for possibly only piecewise continuous coefficient functions $A$ have been developed in Grüne \& Kloeden (2006).

Provided the transition matrix and the invariant projector $P_{+}(t)$ associated with the exponential dichotomy of (2.1) are known, we implemented both Algorithm 3.3 and 4.2 using MATLAB. Here, it is sufficient to restrict to the pseudo-stable manifolds only, since the corresponding pseudo-unstable manifolds are pseudo-stable manifolds of the time-reversed system (2.2), which is given by

$$
\dot{x}=-A(-t) x-F(-t, x) .
$$

Under Hypothesis 2.1 and 2.2 it is defined on $-I$, has the transition operator $\bar{\Phi}(t, s)=\Phi(-t,-s)$ and its linear part admits an exponential dichotomy with $-\beta,-\alpha, K_{-}, K_{+}$and projectors $\bar{P}_{ \pm}(t)=P_{\mp}(-t)$.

Moreover, a couple of further comments are due:

- The quintessence of Theorem 2.3 is that the invariant vector bundles given by the ranges of the dichotomy projectors of (2.1), persist as integral manifolds under perturbation with the nonlinearity $F$. For this reason, it might seem advantageous to replace the initial function $\psi_{0}^{ \pm}(t) \equiv \xi$ by $\psi_{0}^{ \pm}(t)=\Phi(t, \tau) \xi$. However, numerical evidence for this remark is rather small as demonstrated in the example discussed in Subsection 5.1 (Figure 2 (left)).

- The main numerical effort in Algorithm 3.3 and 4.2 is to evaluate the integrals in the definition of the discretized Lyapunov-Perron operators $\tilde{\mathscr{T}}_{T, N}^{ \pm}$and $\tilde{\mathscr{T}}_{h, N}^{ \pm}$, respectively. In order to avoid the discontinuity of Green's function $G(t, s)$ along the diagonal $t=s$, we split the corresponding integrals into two parts and individually apply the following quadrature methods to both integrals:

mid: Composite midpoint rule with $2 N_{n}$ subintervals at recursion depth $n$

trap: Corresponding composite trapezoid rule

quad: MATLAB routine quadv (cf. Gander \& Gautschi (2000)) applied to one subinterval quad $(\mathrm{n})$ : MATLAB integration routine quadv applied to $n$ subintervals

- Since quadrature schemes do not perform well for nonsmooth (or even discontinuous) integrands, we also replaced the piecewise constant iterates $\psi_{n}^{ \pm}$in the recursions (3.6) and (4.4) by piecewise affine continuous functions. We illustrate in Subsection 5.1 (Figure 2 (right)) that this improves our accuracy significantly.

Finally, we remark that the estimate (3.9) for the length $T$ of the truncated integration interval, as well as the radius $\rho>0$ (cf. Remark 2.2(2)) on which the local integral manifolds are defined, and which yields from (2.9), are frequently too pessimistic. As shown below, one might obtain accurate results for smaller values of $T$ and convergence on balls considerably larger than $\rho$. Therefore, we applied our algorithms to (2.2) directly, instead of to the modified problem (2.15).

\subsection{A rotated autonomous example}

Assume that $\alpha, \beta$ are reals with $\alpha<\min \{0, \beta\}$, and let $\omega: \mathbb{R} \rightarrow \mathbb{R}$ be a $C^{1}$-function with bounded derivative. We apply the Lyapunov transform $\Lambda(t):=\left(\begin{array}{cc}\cos \omega(t) & \sin \omega(t) \\ -\sin \omega(t) & \cos \omega(t)\end{array}\right)$ to the planar autonomous sys- 
tem

$$
\left\{\begin{array}{l}
\dot{x}_{1}=\alpha x_{1} \\
\dot{x}_{2}=\beta x_{2}+(2 \alpha-\beta) x_{1}^{2}
\end{array}\right.
$$

and arrive at a nonautonomous ODE of the form (2.2) with

$$
\begin{aligned}
A(t) & =\left(\begin{array}{cc}
\alpha \cos ^{2} \omega(t)+\beta \sin ^{2} \omega(t) & (\beta-\alpha) \cos \omega(t) \sin \omega(t)+\dot{\omega}(t) \\
(\beta-\alpha) \cos \omega(t) \sin \omega(t)-\dot{\omega}(t) & \beta \cos ^{2} \omega(t)+\alpha \sin ^{2} \omega(t)
\end{array}\right) \\
F(t, x) & =(2 \alpha-\beta)\left(\cos \omega(t) x_{1}-\sin \omega(t) x_{2}\right)^{2}\left(\begin{array}{r}
\sin \omega(t) \\
\cos \omega(t)
\end{array}\right)
\end{aligned}
$$

This problem fits well into the framework of Section 2, where the linear part (2.1) has the transition matrix $\Phi(t, s):=\left(\begin{array}{cc}e^{\alpha(t-s)} \cos \omega(t) \cos \omega(s)+e^{\beta(t-s)} \sin \omega(t) \sin \omega(s) & -e^{\alpha(t-s)} \cos \omega(t) \sin \omega(s)+e^{\beta(t-s)} \sin \omega(t) \cos \omega(s) \\ -e^{\alpha(t-s)} \sin \omega(t) \cos \omega(s)+e^{\beta(t-s)} \cos \omega(t) \sin \omega(s) & e^{\alpha(t-s)} \sin \omega(t) \sin \omega(s)+e^{\beta(t-s)} \cos \omega(t) \cos \omega(s)\end{array}\right)$. In particular, we obtain an exponential dichotomy with $\alpha, \beta, K_{ \pm}=1$ and invariant projectors

$P_{+}(t)=\left(\begin{array}{cc}\cos ^{2} \omega(t) & -\cos \omega(t) \sin \omega(t) \\ -\cos \omega(t) \sin \omega(t) & \sin ^{2} \omega(t)\end{array}\right), \quad P_{-}(t)=\left(\begin{array}{cc}\sin ^{2} \omega(t) & \cos \omega(t) \sin \omega(t) \\ \cos \omega(t) \sin \omega(t) & \cos ^{2} \omega(t)\end{array}\right)$.

The specific form of (5.2) yields that its pseudo-stable and -unstable integral manifolds are given by

$$
\hat{S}^{+}=\left\{\left(\tau, \xi, \xi^{2}\right) \in \mathbb{R}^{3}:(\tau, \xi) \in \mathbb{R}^{2}\right\}, \quad \hat{S}^{-}=\left\{(\tau, 0, \eta) \in \mathbb{R}^{3}:(\tau, \eta) \in \mathbb{R}^{2}\right\},
$$

respectively. Therefore, the corresponding integral manifolds of the transformed equation (2.2) read as

$$
\begin{aligned}
& S^{+}=\left\{\left(\tau, \cos \omega(\tau) \xi-\sin \omega(\tau) \xi^{2}, \sin \omega(\tau) \xi+\cos \omega(\tau) \xi^{2}\right) \in \mathbb{R}^{3}:(\tau, \xi) \in \mathbb{R}^{2}\right\}, \\
& S^{-}=\left\{(\tau,-\sin \omega(\tau) \eta, \cos \omega(\tau) \eta) \in \mathbb{R}^{3}:(\tau, \eta) \in \mathbb{R}^{2}\right\}
\end{aligned}
$$

and these nonautonomous sets are visualized in Figure 1. We computed them using Algorithm 3.3 with $\alpha=-1, \beta=1$. Indeed, in the following, we use this toy problem to illustrate Algorithm 3.3 and 4.2.
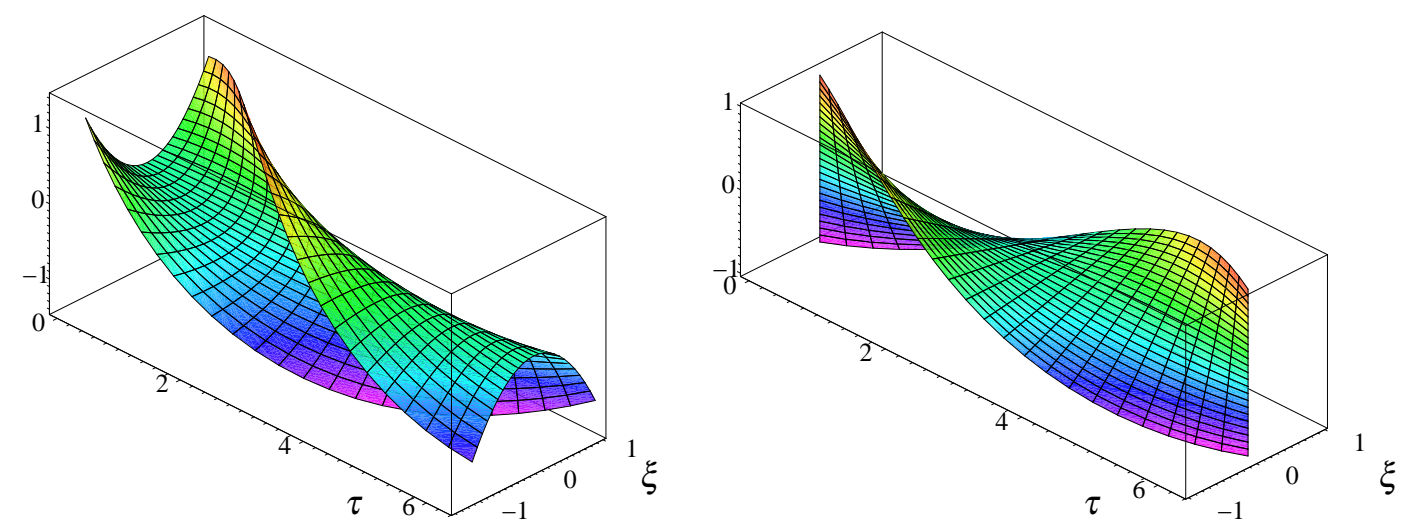

FIG. 1. Pseudo-stable (left) and -unstable (right) integral manifold from Subsection 5.1 for $\omega(t)=t / 2$ and $\tau \in[0,2 \pi]$

First of all, for the nonlinearity $F$, it is not difficult to verify the local Lipschitz estimate

$$
\|F(t, x)-F(t, \bar{x})\| \leqslant 2 \rho|2 \alpha-\beta|\|x-\bar{x}\| \quad \text { for all } t \in \mathbb{R}, x_{1}, \bar{x}_{1} \in[-\rho, \rho], x_{2}, \bar{x}_{2} \in \mathbb{R},
$$


and thus, we can modify $F(t, \cdot)$ outside the set $[-\rho, \rho] \times \mathbb{R}$ such that the above inequality holds globally. To compute the length $T$ of the integration interval according to (3.9), we choose $\rho=\frac{\beta-\alpha}{9|2 \alpha-\beta|}$, get

$$
L=\frac{2}{9}(\beta-\alpha), \quad \ell=2 L=4 \rho|2 \alpha-\beta|=\frac{4}{9}(\beta-\alpha)<\frac{\beta-\alpha}{2}
$$

and set $\sigma=\frac{17}{36}(\beta-\alpha)$. For given accuracy $\varepsilon>0$ and $\|\xi\|<\rho$ these data require an integration interval of lengths $T>\frac{9}{\alpha-\beta} \ln \left(\frac{\varepsilon}{612} \frac{|2 \alpha-\beta|}{\beta-\alpha}\right)$.

We used Algorithm 3.3 to compute the stable fiber bundle of (2.2) for different parameters. Keeping the function $\omega(t)=t / 2, \tau=0$ and the maximal recursion depth 10 fixed, we plotted the number of $F$-evaluations versus the reached relative error for each iteration step. This led to the following results:
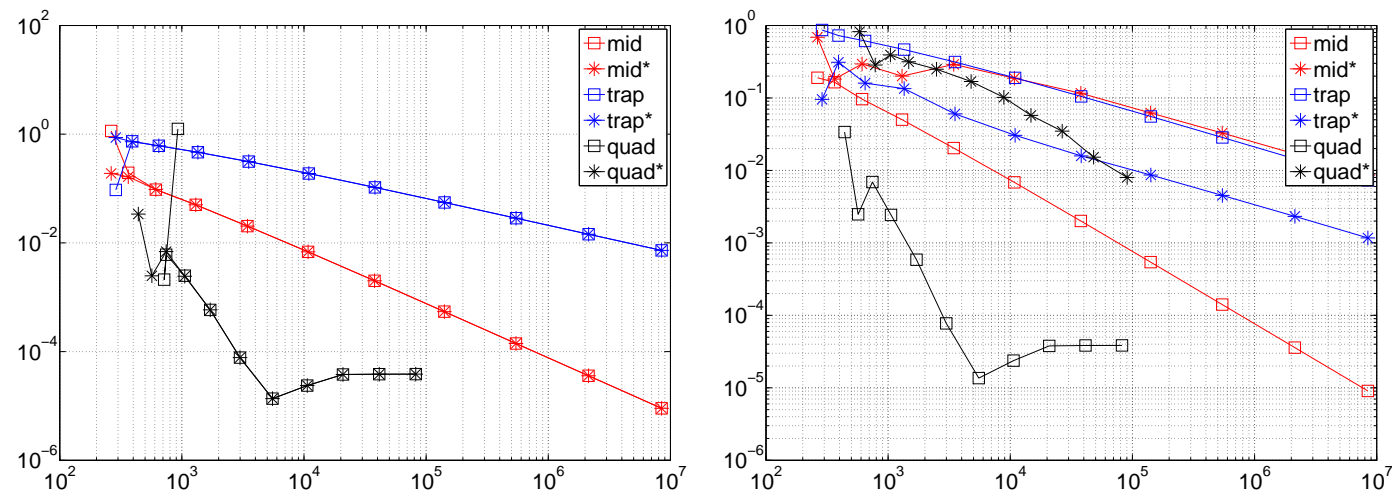

FIG. 2. Subsection 5.1: Relative error vs. number of evaluations in Algorithm 3.3 - Left: Initial function $\psi_{0}^{+}(t) \equiv \xi(\square)$ and $\psi_{0}^{+}(t)=\Phi(t, \tau) \xi(*)$. Right: Piecewise constant $(*)$ or linear integrands $(\square)$

- Figure 2 (left) shows that different initial functions $\psi_{0}^{+}(t) \equiv \xi$ (constant, as suggested in Algorithm 3.3) and $\psi_{0}^{+}(t)=\Phi(t, \tau) \xi$ have minimal influence on the obtained efficiency. The data have been $\xi=(0.5,0), T=10, \alpha=-1, \beta=1, N_{n}=9+2^{n}$, and we used linear interpolation to obtain continuous iterates $\psi_{n}^{ \pm}$.

- On the other hand, Figure 2 (right) illustrates that linear affine continuous functions $\psi_{n}^{+}$yield better results than in the piecewise constant case for integration schemes trap and quad. The data have been $\xi=(0.5,0), T=10, \alpha=-1, \beta=1, N_{n}=9+2^{n}$ and $\psi_{0}^{+}(t)=\Phi(t, \tau) \xi$.

- Figure 3 demonstrates that convergence is generically better in the hyperbolic case $\alpha<0<\beta$ than in the strongly stable situation $\alpha<\beta<0$. Moreover, a slightly larger spectral gap does not necessarily imply better convergence. The data have been $\xi=(0.5,0), T=10, N_{n}=9+2^{n}$, $\psi_{0}^{+}(t)=\Phi(t, \tau) \xi$, and we used linear interpolation.

- From Figure 4 (left), we see that Algorithm 3.3 is quite insensitive under the kind of refinement sequence $N_{n}$. The data have been $\xi=(0.5,0), T=10, \alpha=-1, \beta=1$ and $N_{n}=9+2^{n}$, and we used linear interpolation. For exponential refinement $N_{n}=9+2^{n}$, the recursion depth was 10 (and thus, $N_{10}=1033$ ), in contrast to 32 for polynomial refinement $N_{n}=10+n^{2}$ (and thus, $\left.N_{32}=1034\right)$. 

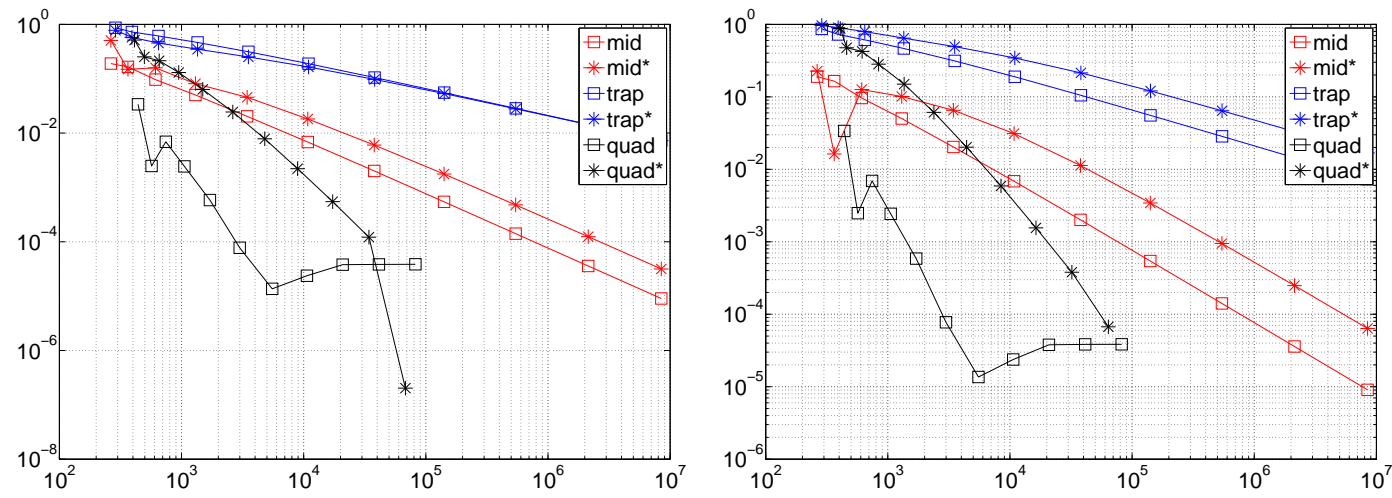

FIG. 3. Subsection 5.1: Relative error vs. number of evaluations in Algorithm 3.3 - Left: $\alpha=-1, \beta=1(\square)$ and $\alpha=-2$, $\beta=-1(*)$. Right: $\alpha=-1, \beta=1(\square)$ and $\alpha=-3, \beta=1(*)$
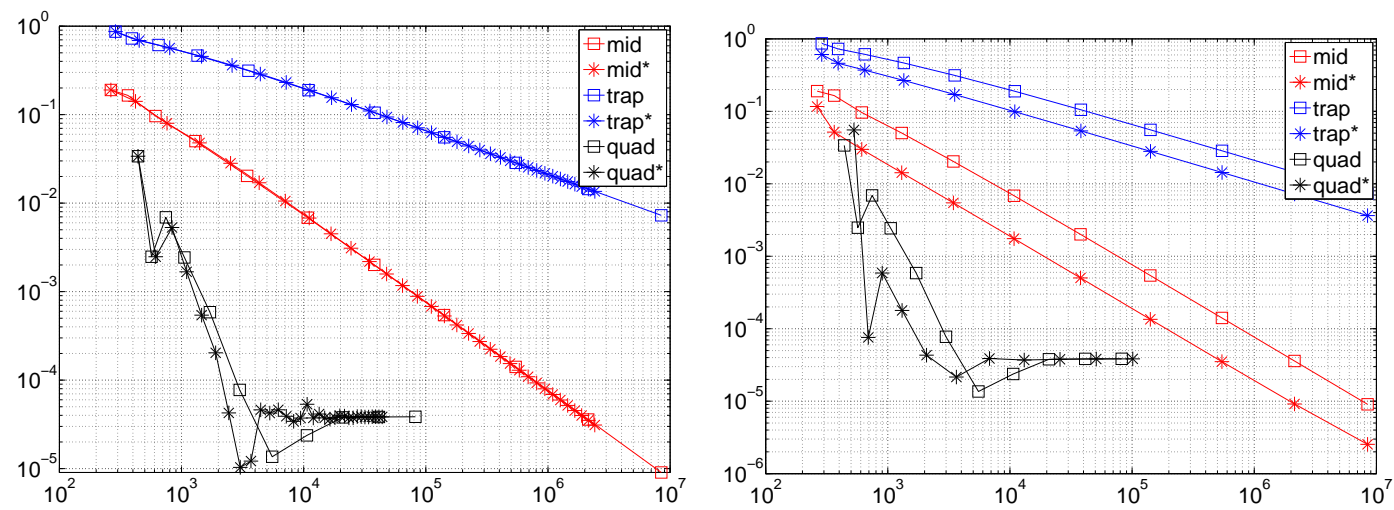

FIG. 4. Subsection 5.1: Relative error vs. number of evaluations in Algorithm 3.3 - Left: $N_{n}=9+2^{n}(\square)$ and $N_{n}=10+n^{2}(*)$. Right: $T=10(\square)$ and $T=5(*)$

- As shown in Figure 4 (right), the performance was better for smaller values of the truncation length $T>0$. Consequently, the estimates for $T$ required from (3.9) are too pessimistic. The data have been $\xi=(0.5,0), \alpha=-1, \beta=1, N_{n}=9+2^{n}$ and $\psi_{0}^{+}(t)=\Phi(t, \tau) \xi$.

- Figure 5 demonstrates that Algorithm 3.3 with an adaptive integration scheme quad is robust and yields convergence for large values of $\xi$ (also in comparison to the corresponding discrete examples discussed in Pötzsche \& Rasmussen (2008)). Here, as predicted in Theorem 3.2, the error grows linearly with $\left\|P_{+}(\tau) \xi\right\|$, whereas the number of evaluations grows logarithmically. We chose $\xi=(0.5,0), T=10, \alpha=-1, \beta=1$ and $N_{n}=9+2^{n}$ and used linear interpolation. 

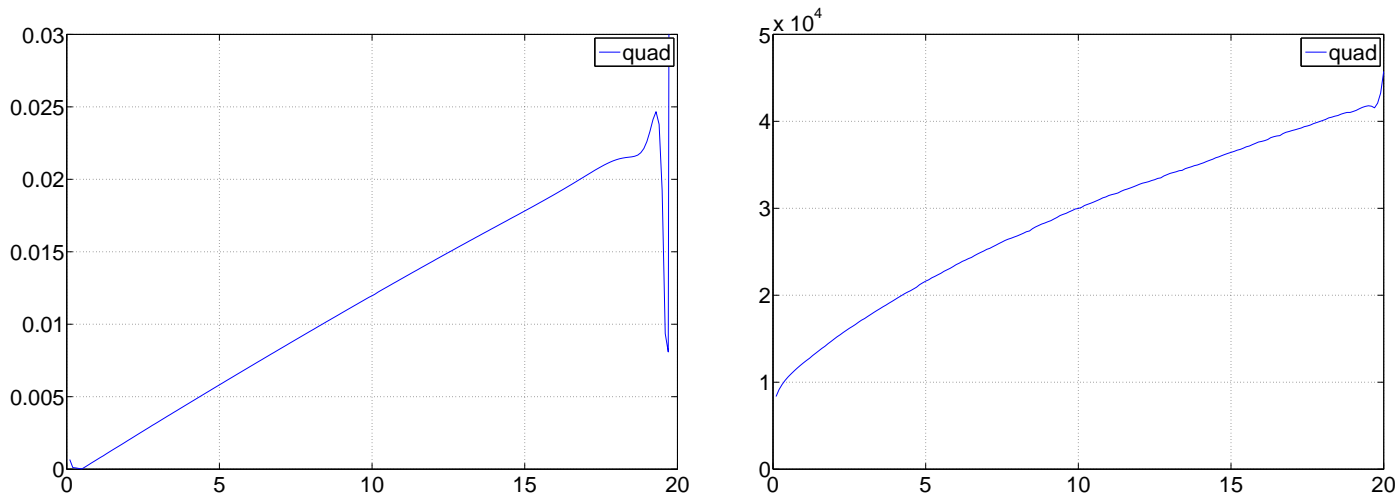

FIG. 5. Subsection 5.1: Performance vs. the argument $\xi \in[1,20]$ in Algorithm 3.3 with quad - Left: $\xi$ vs. the relative error. Right: $\xi$ vs. number of evaluations

\subsection{A nonautonomous test example}

While the above nonautonomous example was continuously differentiable in $t$, we now allow a more general time dependence. Here, suppose that $\alpha, \beta$ are reals with $\alpha<0<\beta$ and $\omega \in \mathscr{L}^{\infty}(\mathbb{R}, \mathbb{R})$. Instead of (5.2), we consider the nonautonomous planar ODE

$$
\left\{\begin{array}{l}
\dot{x}_{1}=\alpha x_{1} \\
\dot{x}_{2}=\beta x_{2}+\left[1+(2 \alpha-\beta)^{2}\right] \omega(t) x_{1}^{2}
\end{array}\right.
$$

which also fits into the framework of Section 2. The general solution for (5.3) is

$$
\varphi(t ; \tau, \xi):=\left(\begin{array}{c}
e^{\alpha(t-\tau)} \xi_{1} \\
e^{\beta(t-\tau)} \xi_{2}+e^{\beta t} e^{-2 \alpha \tau} \xi_{1}^{2}\left[1+(2 \alpha-\beta)^{2}\right] \int_{\tau}^{t} \omega(s) e^{(2 \alpha-\beta) s} \mathrm{~d} s
\end{array}\right) \quad \text { for all } t, \tau \in \mathbb{R}, \xi \in \mathbb{R}^{2} .
$$

Initial pairs $(\tau, \xi) \in \mathbb{R}^{3}$ leading to solutions starting on the stable integral manifold of (5.3) can be obtained from the limit relation $\lim _{t \rightarrow \infty} \varphi(t ; \tau, \xi)=0$, which reduces to the condition

$$
\lim _{t \rightarrow \infty}\left(e^{\beta(t-\tau)} \xi_{2}+e^{\beta t} e^{-2 \alpha \tau} \xi_{1}^{2}\left[1+(2 \alpha-\beta)^{2}\right] \int_{\tau}^{t} \omega(s) e^{(2 \alpha-\beta) s} \mathrm{~d} s\right)=0 .
$$

As in equation (5.2), the unstable integral manifold of (5.3) is $S^{-}=\mathbb{R} \times\{0\} \times \mathbb{R}$ and therefore of little interest for approximation purposes. Yet, our approximation technique is applicable to (5.3) if we set

$$
A(t) \equiv\left(\begin{array}{cc}
\alpha & 0 \\
0 & \beta
\end{array}\right), \quad \quad F(t, x)=\left[1+(2 \alpha-\beta)^{2}\right] \omega(t)\left(\begin{array}{c}
0 \\
x_{1}^{2}
\end{array}\right)
$$

and as in Subsection 5.1, we can verify

$$
\|F(t, x)-F(t, \bar{x})\| \leqslant 2 \rho\left[1+(2 \alpha-\beta)^{2}\right]\|\omega\|_{\infty}\|x-\bar{x}\| \quad \text { for all } t \in \mathbb{R}, x_{1}, \bar{x}_{1} \in[-\rho, \rho], x_{2}, \bar{x}_{2} \in \mathbb{R}
$$

with $\rho=\frac{2}{9}(\beta-\alpha)$; the assumptions of Theorem 2.3 are met for the modified equation (2.15) with

$$
\ell=\frac{4}{9}(\beta-\alpha), \quad \sigma=\frac{17}{36}(\beta-\alpha) .
$$


In case we choose $\omega$ as characteristic function of the interval $[-1,1]$, i.e., $\omega(t)=\chi_{[-1,1]}(t)$, the above limit relation (5.4) yields the explicit representation $S^{+}=\left\{\left(\tau, \xi, s^{+}(\tau, \xi)\right) \in \mathbb{R}^{3}:(\tau, \xi) \in \mathbb{R}^{2}\right\}$ with

$$
s^{+}(\tau, \xi)=\frac{1+(2 \alpha-\beta)^{2}}{2 \alpha-\beta} e^{(\beta-2 \alpha) \tau} \xi^{2} \begin{cases}e^{2 \alpha-\beta}-e^{-(2 \alpha-\beta)}, & \tau \leqslant-1, \\ e^{2 \alpha-\beta}-e^{(2 \alpha-\beta) \tau}, & |\tau| \leqslant-1, \\ 0, \quad \tau \geqslant 1 . & \end{cases}
$$

Having this available, we tested Algorithm 3.3 for the computation of the stable manifold $S^{+}$with $T=10, N_{n}=9+2^{n}$ and different integration schemes. The following table shows the mean absolute error, the mean number of evaluations and the mean difference of the final two iterations over a $31 \times 21$ grid for $\tau \in[-3,2], \xi \in[-1,1]$. Here, for fixed $(\tau, \xi)$ and maximal iteration depth $d$ the difference of the final two iterations is given by the norm of $P_{-}(\tau)\left[\psi_{d}^{+}(\tau)-\psi_{d-1}^{+}(\tau)\right]$ and can serve as criterion to terminate the iteration in Algorithm 3.3.

\begin{tabular}{l|l|rrll} 
method & $d$ & error & evaluations & difference & remark \\
\hline mid & 7 & $4.22 \mathrm{e}-3$ & $1.41 \mathrm{e}+5$ & $5.76 \mathrm{e}-3$ & \\
trap & 7 & $2.74 \mathrm{e}-2$ & $1.42 \mathrm{e}+5$ & $2.51 \mathrm{e}-2$ & \\
quad & 6 & $\mathbf{1 . 0 6 e}-1$ & $4.72 \mathrm{e}+3$ & $2.46 \mathrm{e}-3$ & \\
quad & 7 & $\mathbf{1 . 0 5 e}-1$ & $8.98 \mathrm{e}+3$ & $2.41 \mathrm{e}-4$ & $N_{n}=100+n^{2}$ \\
quad (10) & 4 & $5.85 \mathrm{e}-4$ & $3.37 \mathrm{e}+4$ & $8.14 \mathrm{e}-5$ & \\
quad (10) & 5 & $5.04 \mathrm{e}-4$ & $3.66 \mathrm{e}+4$ & $8.14 \mathrm{e}-5$ & \\
quad (10) & 6 & $4.34 \mathrm{e}-4$ & $4.00 \mathrm{e}+4$ & $7.07 \mathrm{e}-5$ &
\end{tabular}

The poor performance with the routine quad is illustrated in Figure 6 . The adaptive method overlooked the discontinuity of the function $\omega$ at $t=1$ leading to unacceptably large errors (cf. Figure 6 (right)) for $\tau \in[0,1]$. Also a refinement sequence $N_{n}=100+n^{2}$ starting with a larger number of subintervals led to no significant improvement. Yet, this problem was circumvented by subdividing the integration interval
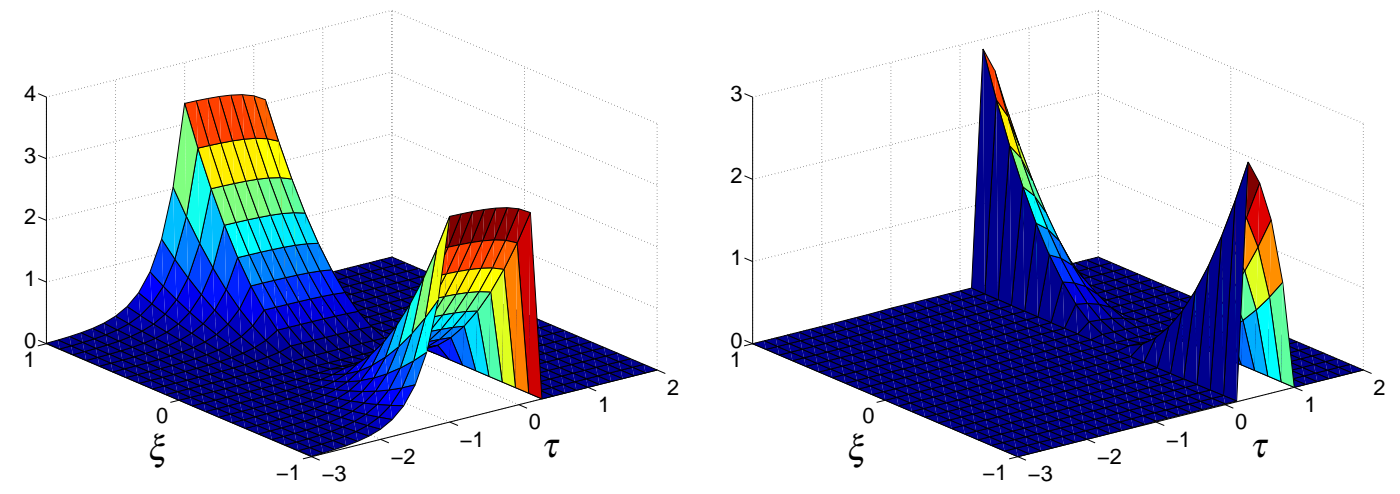

FIG. 6. Erroneous computation of the stable integral manifold from Subsection 5.2 using quad in Algorithm 3.3 with $\alpha=-1$, $\beta=1$ for $\omega(t)=\chi_{[-1,1]}(t), \tau \in[-3,2]$ — Left: stable manifold. Right: absolute error.

to evaluate the integral operator in Algorithm 3.3 into several subintervals and applying quad to each subinterval. We consequently used quad (10), which led to the better results illustrated in Figure 7.

In Figures 8 and 9, we used Algorithm 3.3 with quad (10) and recursion depth 6 to compute the stable manifolds $S^{+}$of (5.3) for hyperbolic growth rates $\alpha=-1, \beta=1$ and different functions $\omega$ : 

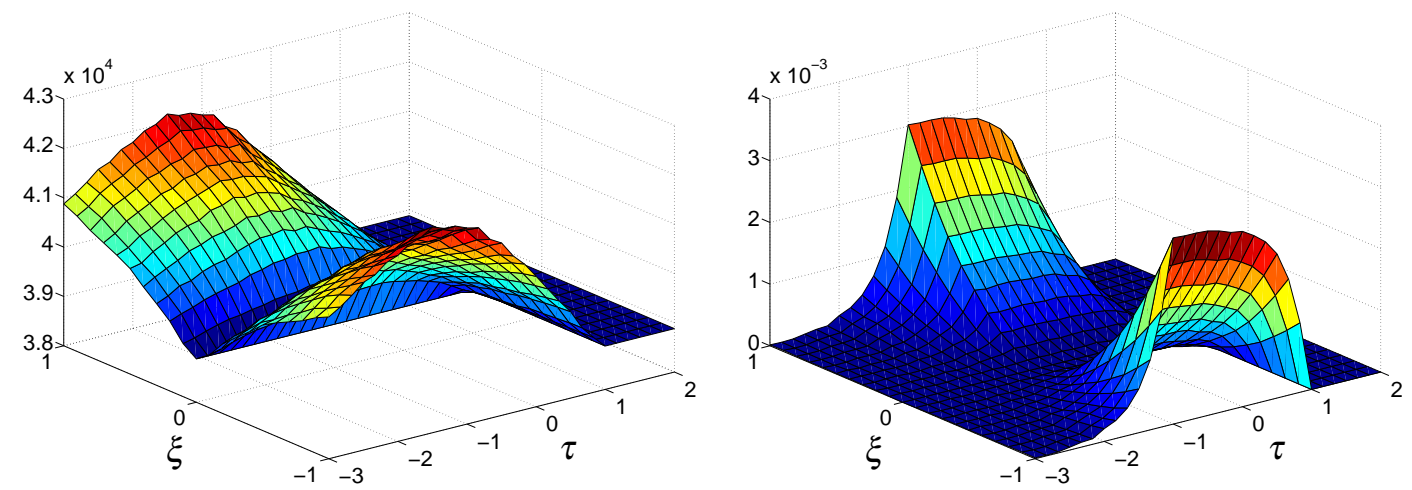

FIG. 7. Number of evaluations (left) and absolute error (right) from Subsection 5.2 computed using quad in Algorithm 3.3 with $\alpha=-1, \beta=1$ for $\omega(t)=\chi_{[-1,1]}(t), \tau \in[-3,2]$.

\begin{tabular}{r|c|rrrl} 
& $\omega(t)$ & $t_{\min }$ & $t_{\max }$ & evaluations & difference \\
\hline discontinuous & $\operatorname{sgn} \sin \left(4 t^{2}\right)$ & 0 & 1.77 & 53715 & $7.84 \mathrm{e}-5(61 \times 41$ grid $)$ \\
discontinuous bump & $\chi_{[-1,1]}(t)$ & -3 & 2 & 40021 & $7.08 \mathrm{e}-5$ \\
discontinuous periodic & $\operatorname{sgn} \sin t$ & $-\pi$ & $\pi$ & 41323 & $1.61 \mathrm{e}-4$ \\
& & $-2 \pi$ & $2 \pi$ & 41299 & $1.59 \mathrm{e}-4$ \\
& $\operatorname{sgn} \sin (2 t)$ & $-\pi$ & $\pi$ & 41985 & $1.43 \mathrm{e}-4$ \\
piecewise constant & $\operatorname{sgn} t$ & -2 & 2 & 40923 & $1.64 \mathrm{e}-4$ \\
smooth transition & $\arctan t$ & -3 & 3 & 40599 & $1.53 \mathrm{e}-4$
\end{tabular}

Finally, we compared Algorithm 3.3 and Algorithm 4.2, as well as different refinement schemes in
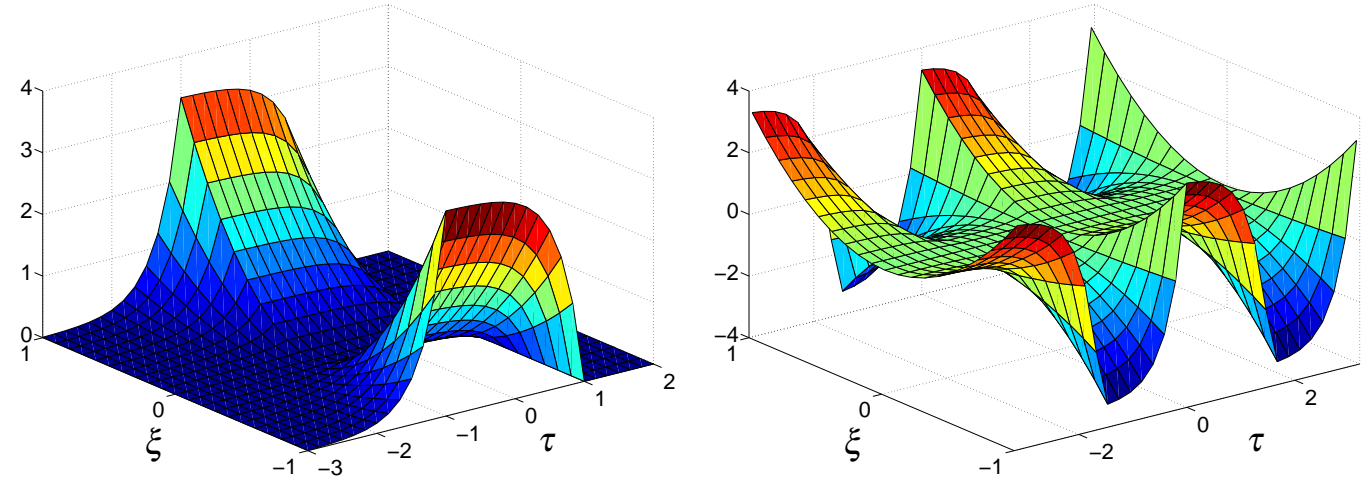

FIG. 8. Stable integral manifold from Subsection 5.2 with $\alpha=-1, \beta=1$ computed using Algorithm 3.3 with quad (10) Left: $\omega(t)=\chi_{[-1,1]}(t), \tau \in[-3,2]$. Right: $\omega(t)=\operatorname{sgn} \sin (2 t), \tau \in[-\pi, \pi]$

Algorithm 4.2, by plotting the relative error versus the number of evaluations for the autonomous version of (5.3) with the parameters $\alpha=-1, \beta=1$ and constant $\omega(t) \equiv-\frac{3}{10}$ on $\mathbb{R}$. In both cases, we computed the value of the stable manifold $s^{+}(\xi)$ at $\xi=1$ with a maximal recursion depth of 10 ; the quadrature method was quad. 

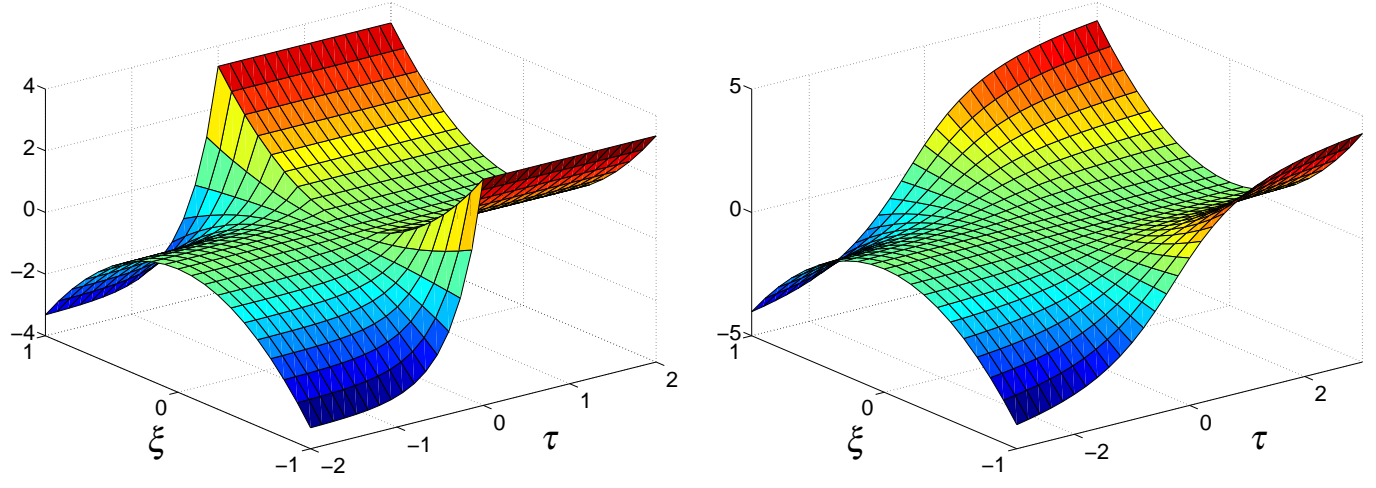

FIG. 9. Stable integral manifold from Subsection 5.2 with $\alpha=-1, \beta=1$ computed using Algorithm 3.3 with quad (10) Left: $\omega(t)=\operatorname{sgn} t, \tau \in[-2,2]$. Right: $\omega(t)=\arctan t, \tau \in[-3,3]$
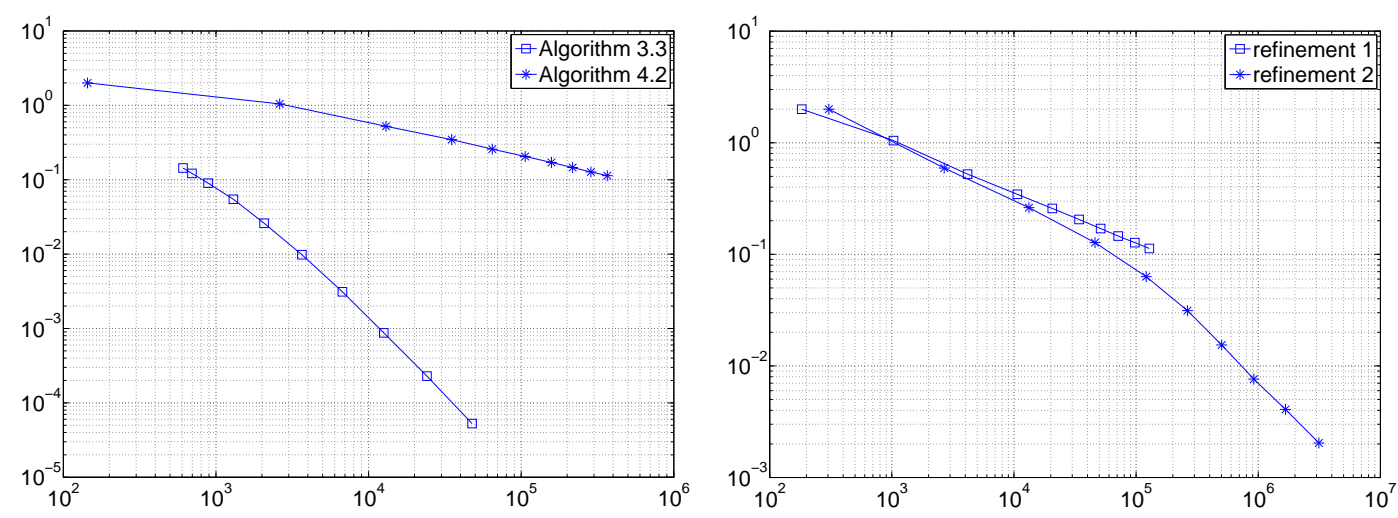

FIG. 10. Subsection 5.2: Computation of $s^{+}(\xi)$ for $\xi=1$ - Left: Algorithm $3.3(\square)$ and Algorithm $4.2(*)$. Right: Algorithm 4.2 with refinement strategies $h_{n}=1 / n, N_{n}=n^{2}(\square)$ and $h_{n}=1 / 2^{n}, N_{n}=n 2^{n}(*)$

- Figure 10 (left) illustrates the performance of Algorithm 3.3 (with $T=10$ and $N_{n}=9+2^{n}$ ) versus Algorithm 4.2 (with refinement $h_{n}:=1 / n$ and $N_{n}:=n^{2}$ ).

- On the other side, Figure 10 (right) shows the results of Algorithm 4.2 for the two refinement strategies $h_{n}:=1 / n, N_{n}:=n^{2}$ and $h_{n}:=1 / 2^{n}, N_{n}:=n 2^{n}$. In the latter strategy we qualitatively follow the requirement (4.5), i.e., $\left(h_{n}\right)_{n \in \mathbb{N}}$ decays exponentially, while $\left(h_{n} N_{n}\right)_{n \in \mathbb{N}}$ grows linearly.

Due to the better performance, we preferred Algorithm 3.3 for our computations in the next subsections.

\subsection{SIRS epidemic model}

The following SIRS model to describe influenza epidemics is adapted from Dushoff et al. (2004). Provided $x_{1}$ denotes the number of susceptible and $x_{2}$ is the number of infected individuals, it is given by 
the nonautonomous planar ODE

$$
\left\{\begin{array}{l}
\dot{x}_{1}=\mu\left(v-x_{1}-x_{2}\right)-\frac{\omega(t)}{v} x_{1} x_{2} \\
\dot{x}_{2}=-\delta x_{2}+\frac{\omega(t)}{v} x_{1} x_{2}
\end{array}\right.
$$

where $\delta^{-1}>0$ is the mean infectious period, $\mu^{-1}$ the average duration of immunity, $v>0$ the total population size and $\omega \in L^{\infty}(\mathbb{R}, \mathbb{R})$ is the time-dependent contact rate. Throughout, we suppose

$$
\operatorname{essinf}_{t \in \mathbb{R}} \omega(t)>\delta
$$

Above all, we have to transform (5.5) into our standard form (2.2). Clearly, $(v, 0)$ is an equilibrium, and we apply the transformation $x \mapsto \Lambda(t)(x-(v, 0))$ to $(5.5)$, where

$$
\Lambda(t):=\left(\begin{array}{cc}
1 & \lambda(t) \\
0 & 1
\end{array}\right), \quad \lambda(t):=\int_{-\infty}^{t}(\mu+\omega(s)) e^{\int_{s}^{t}(\delta-\mu-\omega(r)) \mathrm{d} r} \mathrm{~d} s .
$$

Note that $\lambda$ is the unique bounded solution of the scalar ODE $\dot{x}=(\delta-\mu-\omega(t)) x+\mu+\omega(t)$ (cf. (Aulbach \& Wanner, 1996, Lemma 3.2)) yielding that $\Lambda$ is a Lyapunov transform (meaning that $\Lambda, \Lambda(\cdot)^{-1}$ and $\dot{\Lambda}$ are bounded) and that the transformed equation (5.5) is of the form (2.2) with

$$
A(t) \equiv\left(\begin{array}{cc}
-\mu & 0 \\
0 & -\delta+\omega(t)
\end{array}\right), \quad F(t, x)=\left(x_{1}-\lambda(t) x_{2}\right) x_{2} \frac{\omega(t)}{v}\left(\begin{array}{c}
\lambda(t)-1 \\
1
\end{array}\right) .
$$

Consequently, (2.1) admits an exponential dichotomy on $\mathbb{R}$ with rates $\alpha=-\mu, \beta=\operatorname{essinf}_{t \in \mathbb{R}} \omega(t)-\delta$ and invariant projectors

$$
P_{+}(t)=\left(\begin{array}{cc}
1 & -\lambda(t) \\
0 & 0
\end{array}\right), \quad P_{-}(t)=\left(\begin{array}{cc}
0 & \lambda(t) \\
0 & 1
\end{array}\right) .
$$

It is not difficult to see that the stable integral manifold of (2.2) is $\hat{S}^{+}=\mathbb{R} \times \mathbb{R} \times\{0\}$, and consequently, the stable manifold of $(5.5)$ corresponding to the equilibrium $(v, 0)$ is given by

$$
S^{+}=\left\{(\tau, \xi, v) \in \mathbb{R}^{3}:(\tau, \xi) \in \mathbb{R}^{2}\right\}
$$

Hence, our goal is to compute the unstable manifold $S^{-}$for (2.2) resp. (5.5). Differing from the test examples discussed above, $S^{-}$is not known explicitly.

We worked with growth rates $\mu=1, \delta=1$, a population size $v=1$ and various functions for the time-dependent contact rate $\omega$. In Algorithm 3.3, we fixed $T=10, N_{n}=9+2^{n}$ and tested the above integration schemes. The subsequent experiments show the mean number of evaluations and the mean difference of the final two iterations. Here, $d$ is the maximal iteration depth.

For a constant contact rate $\omega(t) \equiv 2$ (and $\tau \in[-1,1], \xi \in[-0.2,0.2]$ over a $21 \times 21$-grid), we obtained

$$
\lambda(t) \equiv \frac{3}{2}
$$

\begin{tabular}{l|l|rr} 
method & $d$ & evaluations & difference \\
\hline mid & 5 & 10804 & $5.96 \mathrm{e}-5$ \\
trap & 5 & 10952 & $5.21 \mathrm{e}-4$ \\
quad & 6 & 4387 & $4.15 \mathrm{e}-5$ \\
quad (10) & 6 & 36127 & $3.88 \mathrm{e}-5$
\end{tabular}


For piecewise constant $\omega(t) \equiv 2+\frac{1}{2} \operatorname{sgn} t$ (and $\tau \in[-0.5,1], \xi \in[-0.15,0.05], 21 \times 21$-grid), we get

$$
\lambda(t)=\left\{\begin{array}{l}
\frac{5}{3}, \quad t \leqslant 0 \\
\frac{46}{15}-\frac{7}{5} e^{-\frac{5}{2} t}, \quad t>0
\end{array}\right.
$$

\begin{tabular}{l|l|rr} 
method & $d$ & evaluations & difference \\
\hline quad & 6 & 4739 & $1.20 \mathrm{e}-4$ \\
quad & 7 & 9100 & $4.08 \mathrm{e}-5$ \\
quad (10) & 6 & 36070 &
\end{tabular}

Finally, for a $2 \pi$-periodic contact rate $\omega(t) \equiv 2+\frac{1}{2} \sin t$ (and $\tau \in[-\pi, \pi], \xi \in[-0.15,0.1]$ over a $41 \times 21$-grid) it is not possible to compute the function $\lambda$ explicitly. Hence, in order to evaluate the transformation $\Lambda(t)$, we applied a Gauß-Laguerre formula with 20 nodes to approximate

$$
\lambda(t)=\int_{0}^{\infty}(\mu+\omega(t-s)) e^{\int_{t-s}^{t}(\delta-\mu-\omega(r)) \mathrm{d} r} \mathrm{~d} s
$$

\begin{tabular}{l|l|rr} 
method & $d$ & evaluations & difference \\
\hline quad & 6 & 4373 & $2.81 \mathrm{e}-4$ \\
quad (10) & 6 & 36115 & $2.77 \mathrm{e}-4$
\end{tabular}

The unstable integral manifolds for the $\Lambda$-transformed system (5.5) are depicted in Figure 11. Applying the inverse transformation $\Lambda(\cdot)^{-1}$ to these results yields the unstable manifolds for the initial system. The corresponding number of function evaluations is illustrated in Figure 12.
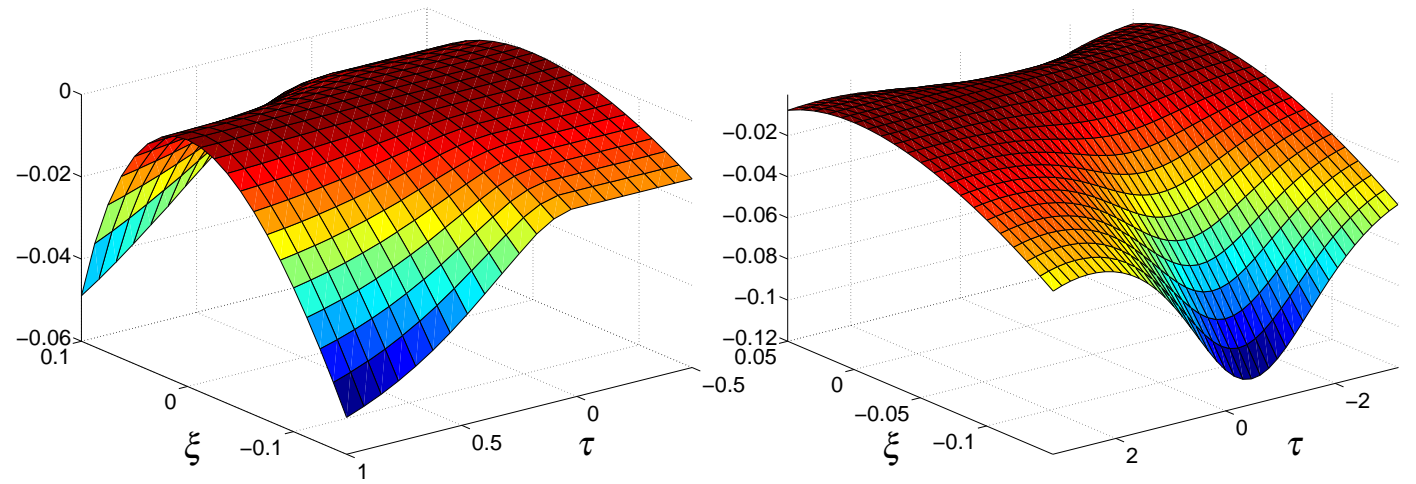

FIG. 11. Unstable integral manifold from Subsection 5.3 with $\mu=1, \delta=1, v=1$ computed using Algorithm 3.3 with quad (10) - Left: $\omega(t) \equiv 2+\frac{1}{2} \operatorname{sgn} t, \tau \in[-0.5,1]$. Right: $\omega(t)=2+\frac{1}{2} \sin t, \tau \in[-\pi, \pi]$

\subsection{Colpitts oscillator}

The following example illustrates our methods using an autonomous ODE, whose right-hand side is globally Lipschitz but not continuously differentiable. The Colpitts oscillator (see, for instance, Maggio et al. (2000)) is one of the most widely used single-transistor circuits to produce sinusoidal oscillations. Mathematically, it is given by a 3-dimensional system

$$
\left\{\begin{array}{l}
\dot{x}_{1}=\frac{1}{\varepsilon}\left(x_{3}-n\left(x_{2}\right)\right) \\
\dot{x}_{2}=\frac{k-1}{\varepsilon} x_{2} \\
\dot{x}_{3}=-\frac{\varepsilon}{k}\left(x_{1}-x_{2}\right)-2 q x_{3}
\end{array}\right.
$$




\section{8 of 30}

\section{Ch. Pötzsche and M. Rasmussen}
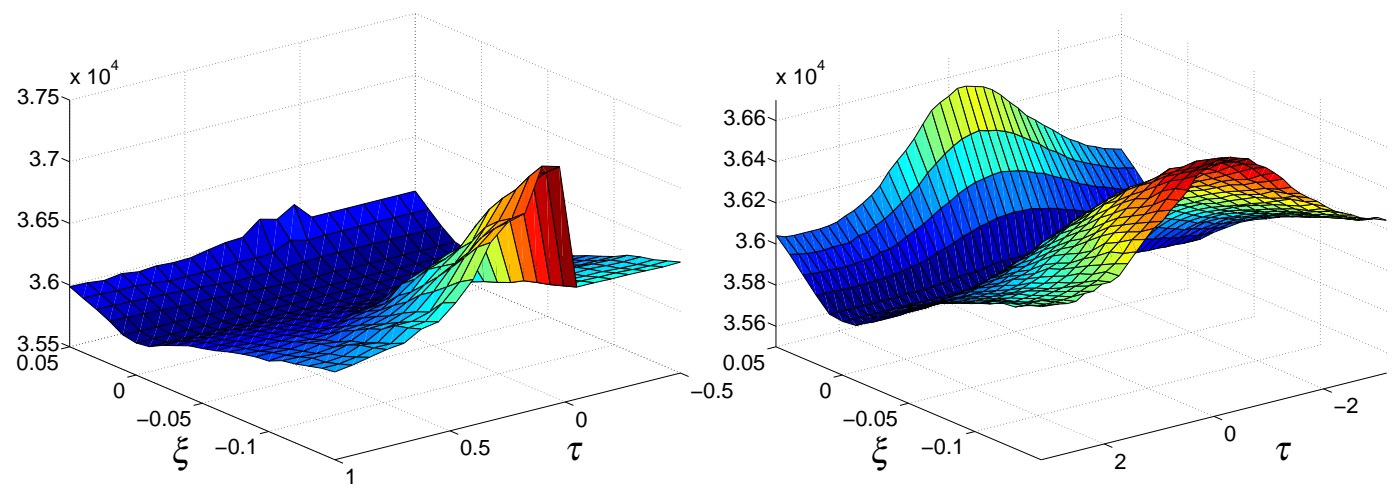

FIG. 12. Function evaluations to compute the unstable integral manifold from Subsection 5.3 with $\mu=1, \delta=1, v=1$ computed using Algorithm 3.3 with quad (10) — Left: $\omega(t) \equiv 2+\frac{1}{2} \operatorname{sgn} t, \tau \in[-0.5,1]$. Right: $\omega(t)=2+\frac{1}{2} \sin t, \tau \in[-\pi, \pi]$

with parameters $\varepsilon, q>0, k>1$ and a piecewise-defined, but continuous nonlinearity

$$
n(x):=\left\{\begin{array}{ll}
-x, & x \leqslant 1 \\
-1, & x>1
\end{array} .\right.
$$

Obviously, (5.6) admits the trivial solution, and we can write it in semilinear form $\dot{x}=A x+F(x)$ with

$$
A:=\left(\begin{array}{ccc}
0 & 0 & \frac{1}{\varepsilon} \\
0 & 0 & \frac{k-1}{\varepsilon} \\
-\frac{\varepsilon}{k} & -\frac{\varepsilon}{k} & -2 q
\end{array}\right), \quad F(x):=-\frac{1}{\varepsilon}\left(\begin{array}{c}
n\left(x_{2}\right) \\
0 \\
0
\end{array}\right)
$$

Since the spectrum of $A$ is given by $\sigma(A)=\{0,-q \pm \omega\}, \omega:=\sqrt{q^{2}-1}$, we know that Assumption 2.1 holds with growth rates $\alpha, \beta$ satisfying $\mathfrak{R}(-q+\omega)<\alpha<\beta<0$, and we can fulfill Assumption 2.2 provided $\frac{2}{\varepsilon}<\Re(q-\omega)$ holds. Thus, equation (5.6) possesses a 2-dimensional stable and a 1-dimensional center manifold. The computation of these manifolds get simplified if we apply the transformation

$$
T:=\left(\begin{array}{ccc}
1 & 1 & -1 \\
k-1 & k-1 & 1 \\
\varepsilon(-q+\omega) & \varepsilon(-q-\omega) & 0
\end{array}\right)
$$

to (5.6), and we arrive at an equivalent system with decoupled linear part

$$
\dot{x}=\left(\begin{array}{ccc}
-q-\omega & & \\
& -q+\omega & \\
& & 0
\end{array}\right) x-\frac{n\left((k-1)\left(x_{1}+x_{2}\right)+x_{3}\right)}{k \varepsilon}\left(\begin{array}{c}
\frac{q+\omega}{2 \omega} \\
\frac{-q+\omega}{2 \omega} \\
1-k
\end{array}\right) .
$$

Due to the autonomous nature, its integral manifolds do not depend on time, i.e., they are invariant manifolds. The Lipschitz-continuous 2-dimensional stable manifold $S^{+}=\left\{\left(\xi, s^{+}(\xi)\right) \in \mathbb{R}^{3}: \xi \in \mathbb{R}^{2}\right\}$, as well as the 1-dimensional center manifold $S^{-}=\left\{\left(s^{-}(\eta), \eta\right) \in \mathbb{R}^{3}: \eta \in \mathbb{R}\right\}$ are depicted in Figure 13. Here, we used parameters $q=2, \varepsilon=8$ and $k=5$.

Let us use the present example (with the same parameters as above) to compare both Algorithm 3.3 and 4.2 with maximal iteration depth $d$ to obtain the following results: 

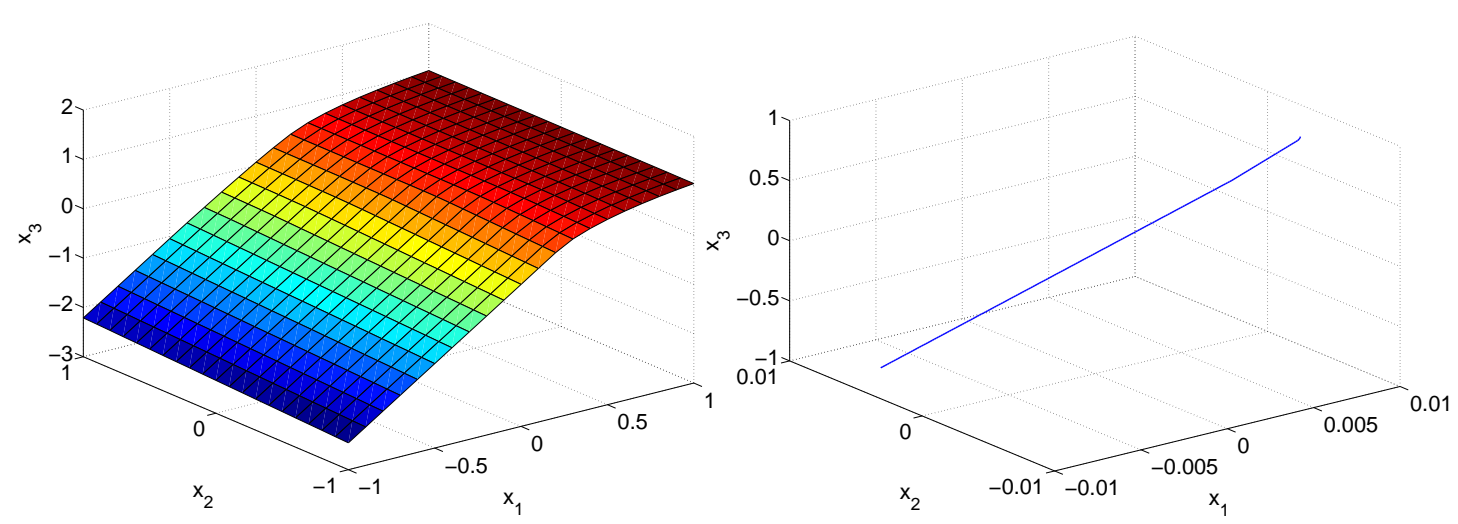

FIG. 13. Stable manifold (left) and center manifold (right) from Subsection 5.4 with $q=2, \varepsilon=8$ and $k=5$.

- Algorithm 3.3: We fixed $T=50, N_{n}=9+2^{n}$ and tested our integration schemes in case of the stable manifold $S^{+}$. The following table contains the mean number of evaluations and the mean difference of the final two iterations over a $41 \times 41$-grid with $\xi \in[-1,1]^{2}$.

\begin{tabular}{l|l|rr}
$S^{+}$ & $d$ & evaluations & difference \\
\hline mid & 7 & 59000 & $1.92 \mathrm{e}-2$ \\
trap & 7 & 60180 & $6.43 \mathrm{e}-3$ \\
quad & 7 & 35770 & $1.36 \mathrm{e}-2$
\end{tabular}

- Algorithm 4.2: This algorithm is not directly applicable for the stable manifold $S^{+}$, since Assumption 4.1 is not fulfilled. However, we used the spectral transformation $y=e^{\kappa t} x$ with $\kappa=0.3$ as described in Remark 4.1 and approximated the 0 -fiber of the transformed system (4.1) which coincides with the stable manifold $S^{+}$. We fixed $h_{n}:=1 / n$ and $N_{n}:=n^{2}, n \in \mathbb{N}$, and the subsequent table shows the mean number of evaluations and the mean difference of the final two iterations over a $41 \times 41$-grid with $\xi \in[-1,1]^{2}$.

\begin{tabular}{l|l|rr}
$S^{+}$ & $d$ & evaluations & difference \\
\hline mid & 7 & 43050 & $1.61 \mathrm{e}-1$ \\
trap & 7 & 43911 & $1.60 \mathrm{e}-1$ \\
quad & 7 & 242913 & $1.63 \mathrm{e}-1$
\end{tabular}

In each case, the number of evaluations lies in the same region; however, the mean difference of the final two iterations is significantly higher for Algorithm 4.2. This underlines the observations from Subsection 5.2, where we have also compared both algorithms. 


\section{REFERENCES}

L. Arnold (1998). Random Dynamical Systems. Springer, Berlin etc.

K. Atkinson (1976). A survey of numerical methods for the solution of Fredholm integral equations of the second kind. SIAM, Philadelphia.

B. Aulbach, M. Rasmussen \& S. Siegmund (2006). 'Invariant manifolds as pullback attractors of nonautonomous differential equations'. Discrete and Continuous Dynamical Systems 15(2):579-596.

B. Aulbach \& T. Wanner (1996). 'Integral manifolds for Carathéodory type differential equations in Banach spaces'. In B. Aulbach \& F. Colonius (eds.), Six Lectures on Dynamical Systems, pp. 45-119. World Scientific, Singapore etc.

F. Colonius \& W. Kliemann (2000). The Dynamics of Control. Birkhäuser, Basel.

L. Dieci \& E. van Vleck (2007). 'Lyapunov and Sacker-Sell spectral intervals'. Journal of Dynamics and Differential Equations 19(2):265-293.

J. Dushoff, J.B. Plotkin, S.A. Levin \& D.J.D. Earn (2004). 'Dynamical resonance can account for seasonality of influenza epidemics'. PNAS 110(48):16915-16916.

M. Fuming \& T. Küpper (1994). 'A numerical method to calculate center manifolds of ODE's'. Applicable Analysis 54:1-15.

W. Gander \& W. Gautschi (2000). 'Adaptive Quadrature Revisited'. BIT 40:84-101.

J. Guckenheimer \& A. Vladimirsky (2004). 'A fast method for approximating invariant manifolds'. SIAM Journal of Applied Dynamical Systems 3(3):232-260.

M. Henderson (2005). 'Computing invariant manifolds by integrating fat trajectories'. SIAM Journal of Applied Dynamical Systems 4(4):832-882.

M. Jolly \& R. Rosa (2005). 'Computation of non-smooth local centre manifolds'. IMA Journal of Numerical Analysis 25:698-725.

M. Jolly, R. Rosa \& R. Temam (2001). ‘Accurate computations on inertial manifolds'. SIAM Journal on Scientific Computing 22(6):2216-2238.

N. Ju, D. Small \& S. Wiggins (2003) 'Existence and computation of hyperbolic trajectories of aperiodically time dependent vector fields and their approximations'. Int. J. Bifurcation Chaos Appl. Sci. Eng. 13(6):1449-1457.

L. Grüne \& P.E. Kloeden (2006). 'Higher order numerical approximation of switching systems'. Syst. Control Lett. 55(9): 746-754.

B. Krauskopf, H.M. Osinga, E.J. Doedel, M.E. Henderson, J. Guckenheimer, A. Vladimirsky, M. Dellnitz \& O. Junge (2005). 'A survey of methods for computing (un)stable manifolds of vector fields'. International Journal of Bifurcation and Chaos 15(3):763-791.

J. Kurzweil (1986). Ordinary Differential Equations. Studies in Applied Mathematics 13. Elsevier, Amsterdam.

D. Liberzon (2003). Switching in Systems and Control. Birkhäuser, Basel.

G. Maggio, M. di Bernardo \& M.P. Kennedy (2000). 'Nonsmooth bifurcations in a piecewise-linear model of the Colpitts oscillator'. IEEE Transactions on Circuits and Systems 47(8):1160-1177.

A. Mancho, D. Small, S. Wiggins \& K. Ide (2003). 'Computation of stable and unstable manifolds of hyperbolic trajectories in two-dimensional, aperiodically time-dependent vector fields'. Physica D 182:188-222.

G. Moore \& E. Hubert (1999). 'Algorithms for constructing stable manifolds of stationary solutions'. IMA Journal of Numerical Analysis 19:375-424.

H. Osinga, G.R. Rokni Lamooki \& S. Townley (2004). 'Numerical approximation of strong (un)stable manifolds'. Dynamical Systems 19(3):195-215.

C. Pötzsche \& M. Rasmussen (2006). 'Taylor approximation of integral manifolds'. Journal of Dynamics and Differential Equations 18(2):427-460.

C. Pötzsche \& M. Rasmussen (2008). 'Computation of nonautonomous invariant and inertial manifolds'.

J. Robinson (2002). 'Computing inertial manifolds’. Discrete and Continuous Dynamical Systems 8(4):815-833. 